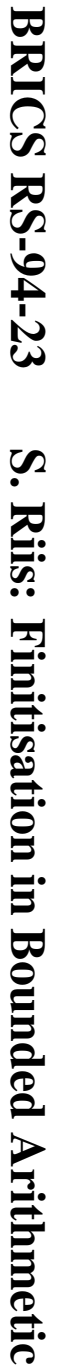

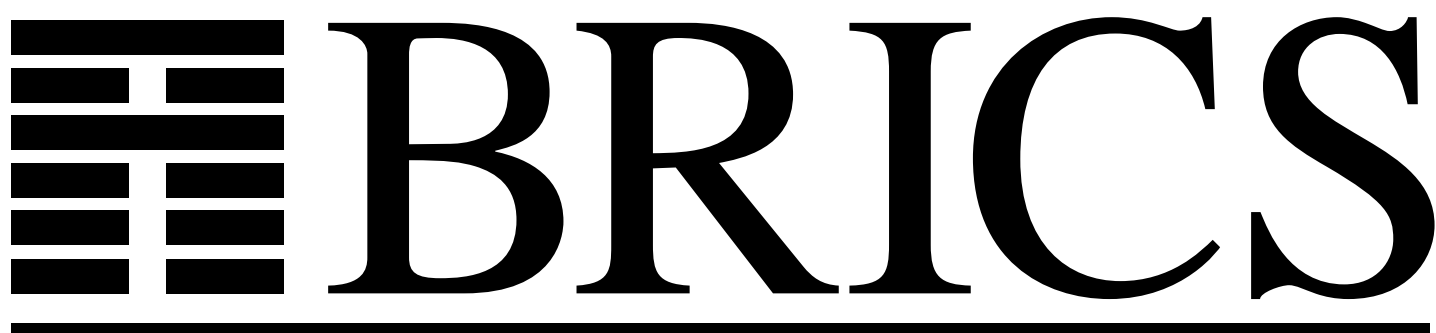

Basic Research in Computer Science

\title{
Finitisation in Bounded Arithmetic
}

Søren Riis 
Copyright (C) 1994, BRICS, Department of Computer Science University of Aarhus. All rights reserved.

Reproduction of all or part of this work is permitted for educational or research use on condition that this copyright notice is included in any copy.

See back inner page for a list of recent publications in the BRICS Report Series. Copies may be obtained by contacting:

\section{BRICS}

Department of Computer Science

University of Aarhus

Ny Munkegade, building 540

DK - 8000 Aarhus C

Denmark

Telephone: +4589423360

Telefax: $\quad+4589423255$

Internet: BRICS@daimi.aau.dk 


\title{
Finitisation in Bounded Arithmetic
}

\author{
Søren Riis* \\ BRICS $^{\dagger}$
}

June 1994

\begin{abstract}
I prove various results concerning undecidability in weak fragments of Arithmetic. All results are concerned with $S_{2}^{1} \subseteq T_{2}^{1} \subseteq S_{2}^{2} \subseteq T_{2}^{2} \subseteq$... a hierarchy of theories which have already been intensively studied in the literature. Ideally one would like to separate these systems. However this is generally expected to be a very deep problem, closely related to some of the most famous open problems in complexity theory.

In order to throw some light on the separation problems, I consider the case where the underlying language is enriched by extra relations and function symbols. The paper introduces a new type of results. These state that the first three levels in the hierarchy (i.e. $S_{2}^{1}, T_{2}^{1}$ and $S_{2}^{2}$ ) are never able to distinguish (in a precise sense) the "finite" from the "infinite". The fourth level (i.e. $T_{2}^{2}$ ) in some cases can make such a distinction. More precisely, elementary principles from finitistical combinatorics (when expressed solely by the extra relation and function symbols) are only provable on the first three levels if they are valid when considered as principles of general (infinitistical) combinatorics. I show that this does not hold for the fourth level.
\end{abstract}

All results are proved by forcing.

\section{Bounded Arithmetic}

The discovery of abstract set theory was like the discovery of the outer space. Set theory provides us with a telescope and has undoubtly affected the general view of the mathematical universe.

\footnotetext{
*This work was initiated in Oxford University England.

${ }^{\dagger}$ Basic Research in Computer Science, Centre of the Danish National Research Foundation.
} 
I view Systems of Bounded Arithmetic as a promising framework of studying the mathematical microcosm. I suggest that questions in complexity theory reside outside the macro-world of ordinary mathematics. I belive that most deeper questions in complexity theory in a strong sense require refined "perception". Many principles which reside deep down in most mathematical arguments, appear equivalent from the normal perspective. (Example: Many elementary counting principles, e.g. the different versions of the elementary pigeon-hole principle). Through a microscope things are perceived quite different. Certain powerful extensions of Bounded Arithmetic could provide such a microscope!

Consistency, not truth, is the right starting point when we consider universal problems. What matters must be deductive powerful viewpoints. Certain extensions of subsystems of Bounded Arithmetic seems to provide a very promising basis for this. This paper is the first in series of planed papers. In these my intension is to isolate more and more powerful, (but unsound) systems of Bounded Arithmetic. Notice that if a universal statement (or more generally a $\Delta_{0}$-statement) $\psi$ is proved from a collection of "false" (in the standard universe $\mathbb{N}$ ) axioms, which are consistent with Bounded Arithmetic, then we know a priori that $\psi$ must actually be true. Because if $\psi$ ware false this would be witnessed in the standard part of each model $\mathbb{M}$ of Bounded Arithmetic, and so according to Gödels completeness theorem this would contradict the consistency assumption. This observation also seems to apply to the conjecture $\mathrm{P} \neq \mathrm{NP}$. To see this recall that $\mathrm{P} \neq \mathrm{NP}$ is equivalent to the statement that "for all programs $\mathcal{P}$, for all $k \in \mathbb{N}$, there exists an input $x$, such that (a) $\mathcal{P}$ uses less than $|x|^{k}$-steps, and either (b1) accept $x$ but $x$ does not satisfies 3 -SAT or (b2) $\mathcal{P}$ does not accept $x$, but $x$ is an instance of 3-SAT". Now if the existential quantifier in "there exists an input $x$ " is bounded by some term $t$, we obtain a $\Delta_{0}$-formula $\Theta$ which implies $\mathrm{P} \neq \mathrm{NP}$. According to the previous remark, if a (consistent) system of Bounded Arithmetic (however unsound) proves $\Theta$, then actually $\mathrm{P} \neq \mathrm{NP}$.

The idea of renouncing central and "obvious" axioms is certainly not new. We recall that there are models in which the self evident parallel postulate of Euclidian Geometry fails, and the wrong principle that each line has many parallel lines-hold true.

\section{Making infinite structures finite}

We are interested in constructing consistent (but unsound) systems of Bounded Arithmetic. In this paper I show that there are fragments of Bounded Arithmetic which have models $\mathbb{M}$ in which any countable structure $S$ (up to elementarily equivalence) 
can be elementarily embedded as a "finite" (in the sense of $\mathbb{M}$ !) structure.

In this section I will illustrate the basic method of this type of result. Consider the impossible ideal that any consistent theory $\Sigma$ has always a finite model. I show that there exists a world in which this ideal is realised. In this world the usual induction axioms only hold for purely existentially defined sets.

Construction: According to the completeness theorem, there is a countable structure $\mathbb{M}$ non-isomorphic, but elementarily equivalent to $\mathbb{N}$, so the same set of $L$ expressible sentences holds in the two structures. There must be an initial segment of $\mathbb{M}$ isomorphic to (and identified with) $\mathbb{N}$. Usually the elements in $\mathbb{N}$ are called standard numbers while the numbers in $\mathbb{M} \backslash \mathbb{N}$ are the so-called "non-standard" numbers.

From an observers perspective outside $\mathbb{M}$ (i.e. from our perspective) there exist "numbers" $n \in \mathbb{M}$ which are infinitely large (i.e $\{1,2, \ldots, n\}$ ) contains infinitely many numbers). However observers inside $\mathbb{M}$ would either not be able to express this, or if we allow them to quantify over second order objects (definable in $\mathbb{M}$ ) they would disagree. These observers of $\mathbb{M}$ would believe that $\{1,2, \ldots, n\}$ was finite simply because it would be finite in their universe $\mathbb{M}$, where they have fewer functions and therefore think more sets are finite! So far all have been folklore. Now the basic part of the argument runs along lines, similar to those in the proof of Theorem 21 in [12].

Suppose $\psi$ states that there exists a bijection from some interval $\{1,2, \ldots, n\}$ to the universe $\mathbb{M}$. From our outside view $\{1,2, \ldots, n\}$ contains infinitely (countable) many numbers, and so our universe must contain a bijection $f$ from $\{1,2, \ldots, n\}$ to $\mathbb{M}$. Suppose now that we actually add a such a map $f$ which maps $\{1,2, \ldots, n\}$ bijectively to $\mathbb{M}$. Suppose also that we extend the language $L$ with an extra function symbol $\bar{f}$ referring to this $f$. Also assume that we add names for each of the countable many elements in $\mathbb{M}$. Let us call this new language $L_{\bar{f}}$. Clearly it is not possible for the model $(\mathbb{M}, f)$ to satisfy the principle of induction. However if $f$ is constructed carefully it turns out that we can force the model to satisfy some amount of induction!

In this example I want to show that one can ensure that $(\mathbb{M}, f)$ satisfies induction for sets which can be existentially defined by $L_{\bar{f}}$-formulas. First take an outside view. The model $\mathbb{M}$ is countable so there are only countably many existentially defined sets $S_{1}, S_{2}, \ldots$ defined by formulas $\psi_{1}(x), \psi_{2}(x), \ldots$ of one free-variable. List these formulas such that each formula appears infinitely many times in the list. At the $k^{\text {th }}$-step in the construction consider the formula $\psi_{k}(x)$, which as an example could be

$$
\exists u \bar{f}(x+13)=2 \cdot u \text {. }
$$

Suppose that in the previous step $f$ has already been defined on a finite set $A \subseteq$ $\{1,2, \ldots, n\}$ with values in $B \subseteq \mathbb{M}$. 
We want the least number principle to be true for the formula $\psi_{k}(x)$. This is done by "forcing" $\psi_{k}(a)$ to be true for the smallest possible $a$, i.e. by letting $f(a+13)$ be even for the smallest value $a$ where this is consistent with the fact that $f$ is a 1-1 map. The conditions $a$ has to satisfy can be expressed in the language $L$ without reference to $\bar{f}$. So in $\mathbb{M}$ we are able to search for such an a by a simple search procedure, which only depends on how $f$ has already been defined on $A$. From an outside view " $<$ " does not well order $\mathbb{M}$, so for a moment we take a look at things from inside $\mathbb{M}$. From this perspective " $<$ " is a well ordering (this is possible because there are fewer sets in $\mathbb{M}$ than in the real universe). So the search procedure must terminate with some output $a$. Observers whether inside or outside $\mathbb{M}$, always agree on first order properties, in this case, whether $a$ actually is the smallest such element.

Now go back to the real world outside $\mathbb{M}$ and proceed to the next step where the formula $\psi_{k+1}(x)$ is considered. Again we force $\psi_{k+1}(a)$ to be true for the smallest possible $a$. Alternatively if we cannot force $\psi_{k+1}(a)$ to be true for any $a$ we know it will never be true (even at doomsday when $f$ is constructed for all formulas).

We must ensure that $f$ eventually defines the required bijection. In the present construction this automatically happens. For instance, for each $a \in\{1,2, \ldots, n\}$ the formula $\psi(x):=\exists y \bar{f}(x)=y \wedge x=a$ eventually forces $a$ to belong to $\{1,2, \ldots, n\}$ (if it does not already do so). The other properties follow for similar reasons.

Now let $\Sigma$ be any consistent theory. According to Skolem-Löwenheims theorem, $\Sigma$ has a countable model $S$. If this model is infinite we assume that $\mathbb{N}$ is the underlying set. If we in the above construction start off by choosing a countable non-standard model $\left(\mathbb{M}, S_{\mathbb{M}}\right)$ elementarily equivalent to $(\mathbb{N}, S)$, we get a model of existential induction in which $\Sigma$ has a finite (in the sense of $\mathbb{M}$ ) model. Thus we have shown:

Proposition 2.0.1 Any consistent theory $\Sigma$ has a model $S$, which is embedded as a finite (=bounded) set in some model $\mathbb{M}$.

Actually suppose that $L$ is a countable language which extend the language of arithmetic, and suppose that $L$ contains undefined relation and function symbols for the language of $\Sigma$. Then the model $\mathbb{M}$ can be chosen such that it satisfies the induction scheme for existential L-formulas.

This shows that any structure $S$, for example structures of strong systems like set theory, can be embedded as "finite" sets in some super-structure. It also shows that we can always assume that a given mathematical domain is "finite" given that our meta-theory (falsely) believes that all sets (and maps etc) in the universe are purely existentially defined.

As the pigeon-hole principle fails for infinite sets, as a corollary we obtain theorem $21[12]:$ 
Corollary 2.0.2 (A.Wilkie, J.Paris) The system $I \exists(f)$ does not prove that $f$ satisfies the pigeon-hole principle.

The results in this paper resemble the ideas just described. However we need to be more careful.

It follows from the main results that countable structures can always be assumed to be (up to elementarily equivalence) finite in certain fragments of Bounded Arithmetic. As we have already indicated this phenomenon is closely related to the fact that the pigeon-hole principle fails heavily in these fragments. And it illustrates the microscope metaphor. One just has to look through the microscope from the right end!

\section{Prelims}

First let me recall some basic notations and facts, essentially all from [4]. Let BASIC denote a finite set of quantifier free formulas relating constants, functions and relations in the first order language $L=L\left(0,1,+, \cdot,|\cdot|, \sharp,\left\lfloor\frac{x}{2}\right\rfloor, \leq,=\right)$. Here $\sharp$ denotes the function given by $a \sharp b=2^{|a| \cdot|b|}$ where $|a|=\left\lceil\log _{2}(a+1)\right\rceil$. An example of a proper choice of BASIC (without coding functions) can be found in [4]. It is convenient to add other functions to the language. We will assume that a function $(w)_{x}$ which takes the value of the $x^{\text {th }}$ element in the sequence coded by $w$ is part of the language. As long as additional functions are polynomially time computable, the results in this section can be stated with no change.

In the first order case atomic formulas are of the form $t=s$ or $t \leq s$ where $s, t$ are terms in $L$, while in the (monadic) second order case additionally, atomic formulas can be of the form $t \in X$ or $X={ }_{2} Y$. (Where "= ${ }_{2}$ " denotes equality between second order variables.)

A first (second) order formula is bounded if all its quantifiers are of the form $\ldots . \forall x \leq t \ldots$ or $\ldots . \exists x \leq t \ldots$. Second order quantifiers are not allowed in bounded formulas. Atomic formulas $X={ }_{2} Y$ are not allowed because they smuggle in an unbounded first order quantifier (Extensionality axiom below).

A first (second) order formula is sharply bounded if it is bounded and all quantifiers are of the form $\ldots . \forall x \leq|t| \ldots$ or $\ldots . \exists x \leq|t| \ldots$.

The class of bounded formulas can be stratified as follows: Let $\Sigma_{0}^{b}=\Pi_{0}^{b}$ be the set of sharply bounded formulas (first or second order formulas depending on the context). Let $\Sigma_{i+1}^{b}\left(\Pi_{i+1}^{b}\right) ; i \geq 0$ be the smallest class of formulas which contains $\Pi_{i}^{b}\left(\Sigma_{i}^{b}\right)$ and is closed under $\wedge, \vee$, sharply bounded quantification, and bounded 
existential quantification (bounded universal quantification). Notice that, except for minor syntactical changes, any bounded formula belongs to some $\Sigma_{i}^{b}$ and to some $\Pi_{i}^{b}$.

Finally let strict- $\Sigma_{i+1}^{b} \subseteq \Sigma_{i+1}^{b}$ denote the set of $\Sigma_{i+1}^{b}$-formulas which are of the form $\exists y_{1} \leq t_{1} \exists y_{2} \leq t_{2} \ldots \exists y_{r} \leq t_{r} \phi$ where $\phi \in \Pi_{i}^{b}$. Similarly, let strict- $\Pi_{i+1}^{b} \subseteq \Pi_{i+1}^{b}$ denote the set of $\Pi_{i+1}^{b}$-formulas which are of the form $\forall y_{1} \leq t_{1} \forall y_{2} \leq t_{2} \ldots \forall y_{r} \leq t_{r} \phi$ where $\phi \in \Sigma_{i}^{b}$.

\subsection{The first order theories}

Let $S_{2}^{i}$ denote the first order theory consisting of BASIC, together with the following "polynomial time" induction scheme, $\left.\varphi(0) \wedge \forall x\left(\varphi\left(\left\lfloor\frac{x}{2}\right\rfloor\right) \Rightarrow \varphi(x)\right) \Rightarrow \forall x \varphi(x)\right)$, where $\varphi \in \Sigma_{i}^{b}$. This scheme is usually denoted by $\Sigma_{i}^{b}$-PIND.

Let $T_{2}^{i}$ denote the first order theory consisting of BASIC together with the $\Sigma_{i}^{b}$ induction scheme, $\varphi(0) \wedge \forall x(\varphi(x) \Rightarrow \varphi(x+1)) \Rightarrow \forall x \varphi(x)$, where $\varphi \in \Sigma_{i}^{b}$. This scheme is usually denoted by $\Sigma_{i}^{b}$-IND.

\subsection{The second order theories}

The (monadic) second order versions of these theories $S_{2}^{i}(\alpha)\left(T_{2}^{i}(\alpha)\right)$ consist of BASIC, $\Sigma_{i}^{b}$-PIND ( $\Sigma_{i}^{b}$-IND) together with the extensionality axiom

$$
\text { EXT : } \forall X, Y\left(X={ }_{2} Y \Leftrightarrow \forall x(x \in X \Leftrightarrow x \in Y)\right)
$$

We do not allow the full comprehension axiom, but follow $[6]$ and equip $S_{2}^{i}(\alpha)\left(T_{2}^{i}(\alpha)\right)$ with the following "NP $\cap$ co-NP" comprehension axiom-scheme: ( $\Delta_{1}^{b}$-comprehension)

$$
\forall x(\varphi(x, \vec{z}, \vec{Z}) \Leftrightarrow \neg \eta(x, \vec{z}, \vec{Z})) \Rightarrow \exists X \forall x(x \in X \Leftrightarrow \varphi(x, \vec{z}, \vec{Z}))
$$

where $\varphi, \eta \in \Sigma_{1}^{b}$.

The underlying logic of these theories is second order predicate logic with second order equality $=_{2}$. It is easy to prove that no deductive strength is lost if $X=_{2} Y$ is taken to be short-hand notation for $\forall z(z \in X \leftrightarrow z \in Y)$, and if EXT and the equality axioms in the underlying logic are dropped.

\subsection{Models of second order theories}

A model of a second order theory $T$ is a pair $(\mathbb{M}, \hat{R})$, where $\hat{R} \subseteq P(\mathbb{M})$, the power set of $\mathbb{M}$, and where $\mathbb{M}$ is a model for the first order part of $T$. The satisfaction relation $\models$ is defined inductively such that second order variables are taken to be the subsets of $\mathbb{M}$ which are in $\hat{R}$. The well known main advantage of using this notion of 
a model, without requiring that $\hat{R}=P(\mathbb{M})$, is that the Compactness Theorem, the Completeness Theorem and Skolem-Löwenheims Theorems hold with minor changes. These facts follow easily (pointed out by A.J.Wilkie in Personal communication) from the natural isomorphism:

Observation 3.3.1 (Transitive collapse) Let $\mathbb{M}^{\triangle}=\mathbb{M}_{\text {num }}^{\triangle} \cup \mathbb{M}_{\text {set }}^{\triangle}$ be a first order model which contains two kinds of elements. One kind $(x, y, z, \ldots)$ denotes numbers and belongs to $\mathbb{M}_{\text {num }}^{\triangle}$, the other kind $(X, Y, Z, \ldots)$ denotes "sets" and belongs to $\mathbb{M}_{\text {set }}^{\triangle}$. If $\tilde{\epsilon}$ is a binary relation on $\mathbb{M}^{\triangle}$, with domain $\mathbb{M}_{\text {num }}^{\triangle}$ and range $\mathbb{M}_{\text {set }}^{\triangle}$ such that

$$
\mathbb{M}^{\triangle} \models(X=Y \Leftrightarrow \forall x(x \tilde{\epsilon} X \Leftrightarrow x \tilde{\epsilon} Y))
$$

then the map $\psi_{\text {collapse }}: X \longrightarrow\left\{x \in \mathbb{M}_{\text {num }}^{\triangle}: \mathbb{M}^{\triangle} \models " x \tilde{\epsilon} X "\right\}$ maps $\mathbb{M}_{\text {set }}^{\triangle}$ bijectively onto a class $\hat{S}$ of subsets of $\mathbb{M}_{\text {num }}^{\triangle}$.

Furthermore $(\mathbb{M}, \hat{S}): \equiv\left(\mathbb{M}_{\text {num }}^{\triangle}, \psi_{\text {collapse }} " \mathbb{M}_{\text {set }}^{\triangle}\right)$ is isomorphic to $\mathbb{M}^{\triangle}$.

From this we get the following version of the Completeness Theorem.

Proposition 3.3.2 $T \cup \mathrm{EXT}$ is consistent if and only if $T$ has a model.

$T \cup$ EXT $\vdash \phi$ if and only if $(\mathbb{M}, \hat{S}) \models \phi$ for all models $(\mathbb{M}, \hat{S}) \models T$.

\subsection{Some special results for $S_{2}^{i}(\alpha)$}

Now I prove that the second order theories $S_{2}^{i}(\alpha)$ are conservative over the corresponding first order theories, in the sense that any model $\mathbb{M} \models S_{2}^{i}$ has an expansion to a model $(\mathbb{M}, \tilde{S}) \models S_{2}^{i}(\alpha)$. A similar result holds for the theories $T_{2}^{i}$. I prove a slightly more general result. Let $\Psi$ be a set of formulas $\psi(x)$, which might contain free second order variables and free first order variables other than $x$.

Definition 3.4.1 By a $\Psi$-substitution scheme $\Sigma$ we will understand a first order formula $\theta_{\Sigma}$ with no second order variables, which contains meta-variables $F_{1}, F_{2}, \ldots, F_{l}$. To each meta-variable $F_{j}$ is associated a term $t_{j}$. A substitution instance $\Sigma(\psi(x)), \psi \in$ $\Psi$ is obtained by replacing each $F_{j}$ in $\theta_{\Sigma}$ with $\psi\left(t_{j}\right)$.

Example 3.4.2 $\Sigma_{i}^{b}(\alpha)$-PIND and $\Sigma_{i}^{b}(\alpha)$-IND are both $\Sigma_{i}^{b}(\alpha)$-substitution schemes.

Definition 3.4.3 In the following let $T(i)=\Sigma+$ BASIC + EXT, where $\Sigma$ is a $\Sigma_{i}^{b}$ substitution scheme. In $T(i)$, BASIC could be any set of first order formulas.

Proposition 3.4.4 If $(\mathbb{M}, \hat{R}) \models T(i)$ there is an expansion $\hat{S} \supseteq \hat{R}$ such that

$$
(\mathbb{M}, \hat{S}) \models T(i)+\Delta_{1}^{b}-\text { comprehension. }
$$


Proof: Assume that $(\mathbb{M}, \hat{R}) \models T(i)$ is a given model. Let

$$
\begin{gathered}
\hat{S}=\left\{S \subseteq \mathbb{M}: \exists \psi_{i}\left(=\psi_{i}\left(x, X_{1}, X_{2}, \ldots, X_{k}\right)\right) \in \Sigma_{1}^{b}, \quad i=1,2\right. \\
\wedge \exists R_{1}, R_{2}, \ldots, R_{k} \in \hat{R} \\
\left.\left(x \in S \Leftrightarrow \psi_{1}\left(x, R_{1}, R_{2}, \ldots, R_{k}\right) \Leftrightarrow \neg \psi_{2}\left(x, R_{1}, R_{2}, \ldots, R_{k}\right)\right)\right\}
\end{gathered}
$$

I claim that $(\mathbb{M}, \hat{S}) \models T(i)+\Delta_{1}^{b}$-comprehension. According to proposition 3.3.2 EXT holds in all second order models, in particular (M, $\hat{S})$. BASIC holds in $(\mathbb{M}, \hat{S})$ because it is a set of first order formulas. It remains to be shown that $(\mathbb{M}, \hat{S}) \models \Sigma$ and to show $(\mathbb{M}, \hat{S}) \models \Delta_{1}^{b}$-comprehension.

Sub claim 1: $(\mathbb{M}, \hat{S}) \models \Sigma(\psi(x))$ for all $\psi \in \Sigma_{i}^{b}$. Notice that if $\psi\left(x, S_{1}, S_{2}, \ldots, S_{k}\right)$ is a $\Sigma_{i}^{b}$-formula with set parameters from $\hat{S}$, there is a $\Sigma_{i}^{b}$-formula $\eta\left(x, R_{1}, R_{2}, \ldots, R_{l}\right)$ with set parameters from $\hat{R}$, such that for all $c \in \mathbb{M}$

$$
(\mathbb{M}, \hat{S}) \models \psi\left(c, S_{1}, S_{2}, \ldots, S_{k}\right) \Leftrightarrow \eta\left(c, R_{1}, R_{2}, \ldots, R_{l}\right) .
$$

Here $\eta$ is obtained from $\psi$ by the following. First, by replacing each appearance of $S_{i}$ with either $\psi_{1}\left(x, R_{1}, R_{2}, \ldots, R_{r}\right)$ or $\neg \psi_{2}\left(x, R_{1}, R_{2}, \ldots, R_{r}\right)$ according to whether $S_{i}$ appears positively or negatively. Second, by bringing it in a "prenex like" form if convenient. Now sub claim 1 follows by noticing that

$$
(\mathbb{M}, \hat{S}) \models \Sigma(\psi) \quad \Leftrightarrow \quad(\mathbb{M}, \hat{S}) \models \Sigma(\eta) \quad \Leftrightarrow \quad(\mathbb{M}, \hat{R}) \models \Sigma(\eta)
$$

and that $(\mathbb{M}, \hat{R}) \models \Sigma(\eta)$ is part of the assumption.

Sub claim 2: $(\mathbb{M}, \hat{S}) \models \Delta_{1}^{b}$-comprehension. Let $\psi_{1}, \psi_{2} \in \Sigma_{1}^{b}$ be given and assume that for some given $a \in \mathbb{M}, \quad(\mathbb{M}, \hat{S}) \models \forall x \leq a\left(\psi_{1}(x) \Leftrightarrow \neg \psi_{2}(x)\right)$. Consider $S=\{x \mid$ $\left.\psi_{1}\left(x, S_{1}, S_{2}, \ldots, S_{k}\right)\right\}$ where $S_{1}, S_{2}, \ldots, S_{k} \in \hat{S}$. As we have already noticed, there are $\Sigma_{1}^{b}$-formulas $\eta_{1}(x, \vec{R})$ and $\eta_{2}(x, \vec{R})$ equivalent to $\psi_{1}$ and $\psi_{2}, S=\left\{x: \eta_{1}(x, \vec{R})\right\}=$ $\left\{x: \neg \eta_{2}(x, \vec{R})\right\} \in \hat{S}$.

Corollary 3.4.5 Every model of first order $S_{2}^{i}, i \geq 1$ has an expansion to a model of $S_{2}^{i}(\alpha)$.

More generally if $U_{1}, U_{2}, \ldots, U_{r}$ are unary relation symbols added to $L$, then every model of $S_{2}^{i}\left(U_{1}, U_{2}, \ldots, U_{r}\right)$, has an expansion to a model of $S_{2}^{i}(\alpha)$

Proof: The first part of the corollary is just the special case where $r=0$. If $r>0$ let $R_{i}:=\left\{x \in \mathbb{M}: \mathbb{M} \models U_{i}(x)\right\}$ and let $\hat{R}:=\left\{R_{1}, R_{2}, \ldots, R_{r}\right\}$. Notice $(\mathbb{M}, \hat{R}) \models S_{2}^{i}(\alpha)-\Delta_{1}^{b}$-comprehension, and use proposition 3.4.4 with $\Sigma$ as the $\Sigma_{i}^{b}$ substitution scheme $\Sigma_{i}^{b}$-PIND.

Notice that Corollary 3.4.5 remains valid if the theories $S_{2}^{i}$ are replaced by $T_{2}^{i}$ for $i \geq 1$. 


\subsection{Some conservation results}

In [5] S.Buss gave a precise characterisation of the $S_{2}^{i}$-provable $\Sigma_{i}^{b}$-definable functions. Actually one of the major justification for dealing with $S_{2}^{i}$ lies in this characterisation. Let me make an observation in this connection. According to a general argument by G.Kreisel the class of provable total recursive functions is always insensitive to the addition of extra universal axioms.

The same argument applies to the class of $\Sigma_{i}^{b}$-definable functions of a theory. The class is insensitive to addition of $\prod_{i}^{b}$-axioms (as long they remain consistent with $S_{2}^{i}$ ). This is because for a theory $T$ in general:

$$
T+\{\forall x \psi(x)\} \vdash \forall x \exists y \theta(x, y) \Rightarrow T \vdash \forall x \exists y \theta(x, y) \vee \neg \psi(y)
$$

which does not produce any new provable total function if $\theta(x, y) \vee \neg \psi(y)$ still is provable equivalent to a $\Sigma_{i}^{b}$-formula. Thus we have

Observation 3.5.1 The class of provable $\Sigma_{i}^{b}$-definable functions of a theory $T$ is immune with respect to the underlying $\Pi_{i}^{b}$-theory.

The following immediately gives us the inclusions

$$
S_{2}^{1}(\alpha) \subseteq T_{2}^{1}(\alpha) \subseteq S_{2}^{2}(\alpha) \subseteq T_{2}^{2}(\alpha) \subseteq \ldots
$$

Proposition 3.5.2 (S.Buss) $S_{2}^{i+1}(\alpha) \vdash T_{2}^{i}(\alpha)$ for all $i \geq 0$.

Proof: Fix an arbitrary $(\mathbb{M}, \hat{S}) \models S_{2}^{i+1}(\alpha)$. First notice that if $X<b$ is a $\Sigma_{i}^{b}$ definable set in $\mathbb{M}$ the convex closure $Y=\operatorname{conv}(X):=\{x<b \mid \exists v \in X \quad v \leq$ $x \wedge \exists u \in X \quad x \leq u\}$ is also a $\Sigma_{i}^{b}$-definable set in $\mathbb{M}$. Let " $\operatorname{dist}\left(Y, Y^{c}\right) \leq d$ " be the $\Sigma_{1}^{b}\left(\Sigma_{i}^{b}\right)$-formula $\phi_{b}(d) \equiv \exists x, y<b(y-x \leq d \wedge y \in Y \wedge x \notin Y)$. By considering the point $\left\lfloor\frac{x+y}{2}\right\rfloor$ obviously $S_{2}^{i+1}(R) \vdash \phi_{b}\left(d\left(\left\lfloor\frac{1}{2}\right\rfloor\right)^{k}\right) \Rightarrow \phi_{b}\left(d\left(\left\lfloor\frac{1}{2}\right\rfloor\right)^{k+1}\right)$ so by $\Sigma_{i+1}^{b}$ - PIND "dist $\left(Y, Y^{c}\right) \leq b " \Rightarrow$ "dist $\left(Y, Y^{c}\right) \leq b\left(\left\lfloor\frac{1}{2}\right\rfloor\right)^{|b|} "$ and by modus ponens and the fact the $S_{2}^{1}(\alpha) \vdash b\left(\left\lfloor\frac{1}{2}\right\rfloor\right)^{|b|}=1$ “dist $\left(Y, Y^{c}\right) \leq 1$ ". As $Y$ is convex, $Y$ has a smallest element in $\mathbb{M}$, and then by definition $X$ has a smallest element. As $\Sigma_{i}^{b}$-LNP $\Leftrightarrow \Sigma_{i}^{b}$-IND we are done.

In some later examples I will use one of the deeper theorems in the subject:

Theorem 3.5.3 (S.Buss) For $i \geq 1, S_{2}^{i+1}(\alpha)$ is $\forall \sum_{i+1}^{b}$-conservative over $T_{2}^{i}(\alpha)$.

Proof: Suppose that $S_{2}^{i+1} \vdash \forall \vec{x} \forall \vec{X} \exists \vec{z} \leq t(\vec{x}) \psi(\vec{x}, \vec{z}, \vec{X})$ where $\psi \in \Pi_{i}^{b}$. It suffices to show that $T_{2}^{i}(\vec{R}) \vdash \exists \vec{z} \leq t(\vec{x}) \psi(\vec{x}, \vec{z}, \vec{R})$, for $\quad \psi \in \Pi_{i}^{b}$.

This follows again by relativising the proof of S.Buss' theorem stating that for any $i \geq 0 \quad S_{2}^{i+1}$ is $\forall^{n d} \Sigma_{i+1}^{b}$-conservative over $T_{2}^{i}$, [7] [5]. 


\section{Some finitisation principles}

One of our aims is to show that there is a fundamental difference between $S_{2}^{2}(\alpha)$ and levels in the hierarchy which are at least as strong as $T_{2}^{2}(\alpha)$. There is a general feeling amongst those working in Bounded Arithmetic that a proof of the non-finite axiomatisability of the first order theory $S_{2}\left(=T_{2}\right)$ would be of great importance. An important step in that direction would be to separate $T_{2}^{1}, S_{2}^{2}$ and $T_{2}^{2}$. These theories are only known to be different under an additional hypothesis from complexity theory.

In [9] $T_{2}^{i}$ and $S_{2}^{i+1}, i \geq 1$ were conditionally separated under the conjectural assumption that the polynomial hierarchy (in complexity theory) does not collapse on level $i+2$. In [8] $S_{2}^{2}$ and $T_{2}^{2}$ were conditionally separated under the conjectural assumption Logspace $\Sigma_{2}^{\Sigma_{2}^{p}} \neq \Delta_{3}^{p}$. However both these assumptions (generally believed to be true) are far beyond current techniques. They both imply $\mathrm{P} \neq \mathrm{NP}$.

The relativised cases $S_{2}^{2}(\alpha)$ and $T_{2}^{2}(\alpha)$ were first separated in [8]. I present new proofs for these relativised cases. This is done by proving the following finitisation principle:

Theorem 4.0.4 If $\psi$ has an infinite model (in the real world), there are structures of $T_{2}^{1}(\alpha)$ in which $\psi$ has a finite (=bounded) model.

I also get the following principle.

Theorem 4.0.5 Suppose that $\theta:=\exists A \psi(A, R)$ is a second order existential statement. Suppose also that $\theta$ is expressed solely by unspecified function and relation symbols. Suppose that $\theta$ has an infinite model (in the real world) where the existential quantifier is not witnessed by any finite set. Then there are structures of $T_{2}^{1}(\alpha)$, in which $\theta$ has a finite model $\{1,2, \ldots, n\}$ where the existential quantifier is not witnessed by any set $A$ of size $\leq \log (n)$.

Since an understanding of the relativised cases, seems to precede an understanding of the unrelativised cases these two results could perhaps be useful in separating $T_{2}^{1}$ from $T_{2}^{2}$ unconditionally.

Finally I prove a finitisation principle which can be used to separate the theories $S_{2}^{1}(\alpha)$ and $T_{2}^{1}(\alpha)$.

Theorem 4.0.6 Suppose that $\psi(\prec, R)$ is a first order statement which is expressed solely by unspecified function and relation symbols. Suppose that in the real world $\psi(\prec, R)$ holds in an infinite model, where $\prec$ defines a total linear ordering. Then there are structures of $S_{2}^{1}(\alpha)$ in which $\psi(\prec, R)$ holds in a finite (=bounded) model and where $\prec$ is the restriction of the order relation $<$. 
These theorems show that when we pass from the real universe to an universe which only contains "feasible" sets, we have the heuristic translations, countable $\rightarrow$ finite, finite $\rightarrow$ poly-logarithmic.

\subsection{Further definitions and assumptions}

Let $L_{2}\left(0,1,+, \cdot f_{\text {fast }}, f_{\text {slow }},=, \leq\right)$ be a second order language where through some basic axioms $f_{\text {fast }}$ and $f_{\text {slow }}$ are ensured to define functions such that $f_{\text {fast }}$ is fastgrowing, and that $f_{\text {slow }}$ is slow growing. Further it is assumed that $f_{\text {slow }}$ is slower than $f_{\text {fast }}$ is fast (!). More precisely assume:

$f_{\text {slow }}, f_{\text {fast }}$ are increasing.

(3) For any fixed $k$, for all sufficiently large $\mathrm{n} f_{\text {slow }}\left(f_{\text {fast }}^{(k)}(n)\right) \leq n$, where

$$
f^{(k)}=\underbrace{f \circ f \circ \ldots \circ f}_{k} .
$$

Definition 4.1.1 A formula $\psi \in L_{2}$ is sharply bounded if each quantifier appears in the context $\exists x \leq f_{\text {slow }}(t)$ or $\forall x \leq f_{\text {slow }}(t)$, where $t \in \operatorname{Term}_{L}$. A formula $\psi \in L_{2}$ is bounded if each quantifier appears in a context $\exists x \leq t$ or $\forall x \leq t$, where $t \in \operatorname{Term}_{L}$.

The class of $\Sigma_{i}^{b}$ formulas and the class of $\Pi_{i}^{b}$ formulas are defined similar to the earlier definition.

Definition 4.1.2 $T^{1}(\alpha)$ denotes the second order theory consisting of a proper base theory Basic together with EXT $+\Delta_{1}^{b}$-comprehension $+\Sigma_{1}^{b}-\mathrm{IND}$.

Given some additional relations $\vec{R}$ we define the first order theory $T^{1}(\vec{R})$ in the obvious way. Notice that if $f_{\text {fast }}(\langle x, y\rangle):=x \sharp y$ and $f_{\text {slow }}(x):=|x|$, for a proper choice of BASIC $T^{1}(\alpha)$ becomes $T_{2}^{1}(\alpha)$. Furthermore notice that in this case

$$
f_{\text {fast }}(n) \leq f_{\text {fast }}(<n, n>)=2^{\left(|n|^{2}\right)}
$$

and therefore $\left|f_{\text {slow }}\left(f_{\text {fast }}^{(k)}(n)\right)\right| \leq\left|f_{\text {slow }}\left(f_{\text {fast }}^{(k)}(<n, n>)\right)\right|=|n|^{2^{k}}$. Clearly for any fixed $k$ and non-standard $n,|n|^{2^{k}}<n$ so the consideration below applies to the theory $T_{2}^{1}(\alpha)$.

Definition 4.1.3 Let $\left\langle x_{1}, x_{2}, \ldots, x_{k}\right\rangle$ be a natural code of the k-tuple. For each $\mathrm{k}$ we introduce quantifiers $\forall^{k}$ and $\exists^{k}$ such that $Q^{k} x \psi(x)$ is shorthand notation for $Q x_{1} Q x_{2} \ldots Q x_{k} \psi\left(<x_{1}, \ldots, x_{k}>\right)$ where $Q \equiv \forall$ or $Q \equiv \exists$. 
Definition 4.1.4 Let $Q^{k} x<t \ldots$ be short-hand notation for $Q x_{1}<t \ldots Q x_{k}<t$, when $\left\langle x_{1}, x_{2}, \ldots, x_{k}\right\rangle=x$. Let $\theta(\vec{x})$ be a formula in some relational language $L\left(R_{1}, R_{2}, \ldots, R_{l}\right)$. For a relation symbol $\mathrm{S}$, the formula $\theta_{S}(\vec{x})$ denotes the formula which appears if each quantifier in $\theta$ is restricted to S. By $\theta_{<a}(\vec{x})$ we understand the formula which appears by restricting each quantifier in $\theta$ to $[0, a)$.

\subsection{A version of the completeness theorem}

As I have already pointed out, in the real mathematical universe it is not true that any finite consistent set of first-order sentences has a finite model. But there are $T_{2}^{1}(\alpha)$-universes where such a strong form of the completeness theorem holds.

Theorem 4.2.1 (Finitisation principle) Let $\theta(\vec{R})$ be a first order property expressed in some relational language $\tilde{L}=L(\vec{R})$. Suppose that $\eta(n)$ is an arithmetical first order property expressible in the language $L$ of arithmetic and suppose that $\eta(n)$ holds for arbitrary large $n$. If $\theta(\vec{R})$ has a model then the theory $T^{1}(\vec{R})+\exists n \eta(n) \wedge \theta_{<n}(\vec{R})$ has a model.

First we shall make some preparation for the proof. Let $\tilde{L}$ and $\theta(\vec{R})$ be given as in the theorem. We can assume that all relations are $r$-ary. $\theta(\vec{R})$ is assumed to have an infinite model, so by Skolem-Löwenheims Theorem, we can assume $\theta(\vec{R})$ has an infinite countable model $S_{s t}$ on a subset of the natural numbers. Furthermore, we can assume $S_{s t}$ is a model with an underlying co-countable set, and if convenient, that an extra unary relation symbol denoting membership of $S_{s t}$ is added to the language.

Let $(\mathbb{M}, S)$ be a countable non-standard model for the language $\tilde{L}(R):=L \cup \tilde{L}$ which is elementarily equivalent to the standard model $\left(\mathbb{N}, S_{s t}\right)$. Use overspill to pick a non-standard number $n$ such that $\mathbb{M} \models \eta(n)$.

Fix a non-standard number $b_{0}<n$ such that $b_{0}^{k}<n$ for each standard number $k$, and such that $f_{\text {slow }}\left(f_{\text {fast }}^{(k)}(n)\right)<b_{0}$ for every standard number $k$. Use overspill to pick $c \in \mathbb{M}$ non-standard such that $f_{\text {slow }}(c)<b_{0}$ and such that $c>f_{\text {fast }}^{(k)}(n)$ for all standard numbers $k$.

Definition 4.2.2 Let $\mathcal{P}_{k}, k \in \omega$, be the set of all partial (1-1)-maps $\alpha$ which have $\operatorname{dom}(\alpha) \subseteq\{1,2, \ldots, c\}$ and $\operatorname{ran}(\alpha) \subseteq \mathbb{M}$ such that $|\operatorname{dom}(\alpha)| \leq b_{0}^{k}$, and such that $\alpha$ maps points in $[0, n)$ to $S$, and maps points in $\{n+1, n+2, \ldots, c\}$ to $\mathbb{M} \backslash S$.

Let $\mathcal{P}=\cup_{k \in \omega} \mathcal{P}_{k}$ and let $(\mathcal{P}, \subseteq)$ be $\mathcal{P}$ ordered under inclusion. 


\subsection{Generic maps}

Let us now make a few basic definitions:

Definition 4.3.1 $\mathcal{D} \subseteq \mathcal{P}$ is called dense if $\forall \alpha \in \mathcal{P} \exists \beta \in \mathcal{D}$ such that $\beta \supseteq \alpha$.

A subset $S \subseteq \mathbb{M}$ is quasi-definable in $\mathbb{M}$ if there is $\theta(x) \in L\left(R_{\omega}\right)$ such that $S=\{x \in M:(\mathbb{M}, \omega) \models \theta(x)\}$ where $R_{w}$ is interpreted by $\omega$. We allow $\theta$ to contain parameters from $\mathbb{M}$.

Notice that in [1] the similar notion (just called definable) is $S=\{x \in M \mid \exists n \in$ $\left.R_{w} \theta(x, n)\right\}$, which would not work in our case because we cannot force formulas in general to be equivalent to existential formulas. It should also be noticed that any extension of the notion of quasi-definability which does not produce uncountably many quasi-definable dense subsets of $\mathcal{P}$, would work.

Example 4.3.2 The set $\mathcal{P}$ is quasi-definable. The initial segment $\left[0, b_{0}^{\omega}\right)$ is quasidefinable.

Definition 4.3.3 $\mathcal{G} \subseteq \mathcal{P}$ is a generic filter if

$$
\begin{aligned}
& \forall \alpha, \beta \in \mathcal{G} \exists \gamma \in \mathcal{G} \gamma \supseteq \alpha \wedge \gamma \supseteq \beta . \\
& \forall \alpha \in \mathcal{G} \forall \beta \in \mathcal{P} \alpha \supseteq \beta \Rightarrow \beta \in \mathcal{G} . \\
& \mathcal{G} \cap \mathcal{D} \neq \emptyset \quad \text { for each quasi-definable dense set } \mathcal{D} \subseteq \mathcal{P} .
\end{aligned}
$$

Lemma 4.3.4 For every $\alpha_{0} \in \mathcal{P} \exists \mathcal{G} \subseteq \mathcal{P}$ generic such that $\alpha_{0} \in \mathcal{G}$.

Proof: $\mathbb{M}$ is assumed to be countable so that there is at most countably many quasidefinable sets $\mathcal{D} \subseteq \mathcal{P}$. List those as $\mathcal{D}_{1}, \mathcal{D}_{2}, \mathcal{D}_{3}, \ldots$. Pick $\alpha_{1} \supseteq \alpha_{0}$ such that $\alpha_{1} \in \mathcal{D}_{1}$, pick $\alpha_{2} \supseteq \alpha_{1}$ such that $\alpha_{2} \in \mathcal{D}_{2}$, etc. and let $\mathcal{G}:=\left\{\beta \in \mathcal{P} \mid \exists j \in \omega \quad \beta \subseteq \alpha_{j}\right\}$. It is straightforward to check that $\mathcal{G}$ is generic.

Definition 4.3.5 $\tilde{\alpha} \subseteq \mathbb{M}$ is generic if there is a generic $\mathcal{G} \subseteq \mathcal{P}$ such that $\tilde{\alpha}=\cup_{\alpha \in \mathcal{G}} \alpha$.

\subsection{Sketch of proof}

Now let me sketch the proof. We are given a non-standard model $(\mathbb{M}, S) \equiv_{e}\left(\mathbb{N}, S_{s t}\right)$ in which the "structure" $S$ we want to miniaturise is a part. We can assume that the language contains the language of arithmetic together with extra relations, denoting the relations in $S$. 
First, it is shown that some (actually any) generic $\tilde{\alpha}$ is a bijection from $[0, c)$ to $\mathbb{M}$, mapping $[0, n)$ onto $S$. At this stage we also have to show certain lemmas about the forcing relation in order to ensure it behaves well.

Second, it is shown that for some (actually any) generic map $\tilde{\alpha}, \quad(\mathbb{M}, \tilde{\alpha}) \models$ $\exists^{*}$-LNP, where $\exists^{*}$-LNP denotes the least number principle for formulas in which all quantifiers are either existential (positive appearance) or are restricted to $\left[0, b_{0}\right)$. This part of the argument is based on the same idea as the proof of theorem 21 in [12] (see also the introduction).

Third, it is noticed that the constants, relations and functions in the miniaturised structure $S_{\text {mini }}:=\tilde{\alpha}^{-1}(S)$ are $\exists \cap \forall$-definable in the generic model $(\mathbb{M}, \tilde{\alpha})$.

Fourth, it is shown that each formula expressing a $\Sigma_{1}^{b}$ - property about the miniaturised model and numbers in $[0, c)$, can be translated into an $\exists^{*}$-formula. This ensures that LNP holds for $\Sigma_{1}^{b}$-formulas with parameters in $[0, c)$.

Finally a concrete model $\mathbb{M}^{*}$ is constructed as the smallest initial segment of $[0, c)$ which contains $[0, n)$ and is closed under $f_{\text {fast }}$.

\subsection{The forcing relation}

First we need to show some basic fact about generic maps. For simplicity we reduce logical constants. So suppose that $\forall: \equiv \neg \exists \neg$ and $\wedge: \equiv \neg \vee \neg$. Extend the language with names for the elements in $\mathbb{M}$. Also extend the language $\tilde{L}(R)$ by an extra binary relation symbol $\bar{\alpha}$. For sentences in this language $\tilde{L}(\vec{R}, \bar{\alpha})$ we define the forcing relation inductively as follows:

$\alpha \Vdash \psi$ if $\psi$ does not contain $\bar{\alpha}$, is atomic and true.

$$
\begin{gathered}
\alpha \Vdash \bar{\alpha}(a, b) \quad \text { iff } \quad \alpha(a) \text { is defined and equals } b . \\
\alpha \Vdash \psi \vee \eta \quad \text { iff } \quad \alpha \Vdash \psi \text { or } \alpha \Vdash \eta . \\
\alpha \Vdash \exists x \psi(x) \quad \text { iff for } \underline{\text { some }} a \in \mathbb{M} \quad \alpha \Vdash \psi(a) .
\end{gathered}
$$

The forcing relation for negation satisfies:

$$
\alpha \Vdash \neg \psi \text { iff for no } \beta \supseteq \alpha, \beta \in P \beta \Vdash \psi \text {. }
$$

\subsection{Soundness of the forcing relation}

We have to make sure that the forcing relation satisfies certain key properties. Except for lemma 4.6.4 below, the reader who is familiar with forcing techniques could ignore this section. 
Definition 4.6.1 Let $\Sigma\left(b_{0}\right)$ be the set of formulas where all quantifiers are restricted to $\left[0, b_{0}\right)$.

Notice $f_{\text {slow }}(c)<b_{0}$ and therefore in the final model all sharply bounded quantifiers are restricted to $\left[0, b_{0}\right)$.

Lemma 4.6.2 (Forcing lemma) The forcing relation has the following properties: Extension property: If $\alpha \Vdash \psi$ and $\beta \supseteq \alpha$ then $\beta \mid \vdash \psi$

Consistency: For no $\alpha \in \mathcal{P}$ and for no $\psi$, does both $\alpha \Vdash \psi$ and $\alpha \Vdash \neg \psi$ hold.

Completeness: For each generic set $\mathcal{G} \subseteq \mathcal{P}$, and for each $\psi$ there is $\alpha \in \mathcal{G}$ such that $\alpha \Vdash \psi$ or $\psi \Vdash \neg \psi$.

Soundness 1: If for a generic map $\tilde{\alpha},(\mathbb{M}, \tilde{\alpha}) \models \psi$, there is $\alpha \in \mathcal{G}$ such that $\alpha \Vdash \psi$. Soundness 2: If $\alpha \Vdash \psi$ then $(\mathbb{M}, \tilde{\alpha}) \models \psi$ for any generic $\tilde{\alpha} \supseteq \alpha$.

Proof: Extension property: First notice that the claim holds for atomic formulas. Clearly the extension lemma holds if $\psi \equiv \psi_{0} \vee \psi_{1}$, or if $\psi \equiv \exists y \psi_{0}(y)$. Suppose $\psi \equiv \neg \psi_{0}, \alpha \vdash \psi$, and $\beta \supseteq \alpha$ is given. By definition for no $\alpha^{\prime} \supseteq \alpha, \alpha^{\prime} \vdash \psi_{0}$. The ordering $\supseteq$ of the forcing conditions $\mathcal{P}$ is transitive for no $\alpha^{\prime} \supseteq \beta, \alpha^{\prime} \Vdash \psi_{0}$. By definition $\beta \mid \vdash \psi$.

Consistency: Direct by the inductive definition.

Completeness: Let $\mathcal{D}:=\{\alpha: \alpha \Vdash \psi \vee \neg \psi\}$. Notice $\mathcal{D}$ is quasi-definable and dense so there is $\alpha_{0} \in \mathcal{G} \cap \mathcal{D}$.

By definition either $\alpha_{0} \Vdash \psi$ or $\alpha_{0} \Vdash \neg \psi$.

Soundness $1+2$ : Both claims are proved simultaneously using induction on the number of logical constants in $\psi$. The case where $\psi$ is atomic is straightforward, and so is the case where $\psi \equiv \psi_{0} \vee \psi_{1}$ or $\psi \equiv \exists y \psi_{0}(y)$.

If $(\mathbb{M}, \tilde{\alpha}) \models \neg \psi$, by induction there cannot be $\alpha \in \mathcal{G}$ such that $\alpha \Vdash \psi$. By completeness there is $\alpha \in \mathcal{G}$ such that $\alpha \Vdash \neg \psi$.

If $\alpha \vdash \neg \psi$ but $(\mathbb{M}, \tilde{\alpha}) \models \psi$ for some generic map $\tilde{\alpha} \supseteq \alpha$, there is $\beta \in \mathcal{G}$ such that $\beta \Vdash \psi$. By definition $\alpha$ and $\beta$ have a common extension in $\mathcal{P}$. By use of the extension and the consistency property we get a contradiction.

Corollary 4.6.3 Any generic $\tilde{\alpha}$ is a bijection from $[0, c)$ to $\mathbb{M}$, which maps $[0, n)$ onto $S$.

Proof: Let $a \in[0, c]$ be an arbitrary element. Notice that $\mathcal{D}_{a}:=\{\alpha: \alpha(a)$ is defined $\}$ is both dense and quasi-definable. By definition there is $\alpha_{0} \in \mathcal{D} \cap \mathcal{G}$.

As $\alpha_{0} \subseteq \tilde{\alpha}$ this shows that $\tilde{\alpha}$ has domain $[0, c)$. The other properties are proved by a similar argument. 
Lemma 4.6.4 For each $\psi(\vec{x}) \in \Sigma\left(b_{0}\right)$ there is $k \in \omega$ which does not depend on the parameters in $\psi(\vec{x})$ and $\mathbb{M}$-definable maps $\vec{x} \hookrightarrow V_{\vec{x}}^{D}$ and $\vec{x} \hookrightarrow V_{\vec{x}}^{R}$ such that

For all $\alpha \in \mathcal{P}$ with $\operatorname{Dom}(\alpha) \supseteq V_{\vec{x}}^{D}$ and $\operatorname{Ran}(\alpha) \supseteq V_{\vec{x}}^{R}$.

$$
\alpha \Vdash \psi(\vec{x}) \text { or } \alpha \Vdash \neg \psi(\vec{x}) \text {. }
$$

$$
\text { For all } \alpha \in \mathcal{P}, \alpha_{\mid V_{\vec{x}}^{D} \cup V_{\vec{x}}^{R}} \mid \vdash \psi(\vec{x}) \text { if } \alpha \Vdash \psi(\vec{x}) \text {. }
$$

Proof: If $\psi(x)$ is atomic and is of the form $\alpha(u, v), \alpha(x, v), \alpha(u, x)$ or $\alpha\left(x_{1}, x_{2}\right)$ let $V_{x}^{D}:=\{u\}, V_{x}^{D}:=\{u\}, V_{x}^{D}:=\{v\}$ or $V_{x_{1}, x_{2}}^{D}:=\left\{x_{1}\right\}$, and similar let $V_{x}^{R}:=\{v\}$, $V_{x}^{R}:=\{v\}, V_{x}^{R}:=\{v\}$ and $V_{x_{1}, x_{2}}^{R}:=\left\{x_{2}\right\}$.

If $\psi \equiv \psi_{0} \vee \psi_{1}$ let $V_{\vec{x}}^{D}:=V_{\vec{x}}^{D, 0} \cup V_{\vec{x}}^{D, 1}$, and let $V_{\vec{x}}^{R}:=V_{\vec{x}}^{R, 0} \cup V_{\vec{x}}^{R, 1}$. If $\psi \equiv \exists u \leq$ $b_{0} \psi_{0}(u, \vec{x})$ let $V_{\vec{x}}^{D}:=\bigcup_{u \leq b_{0}} V_{u, \vec{x}}^{D, 0}$ and $V_{\vec{x}}^{R}:=\bigcup_{u \leq b_{0}} V_{u, \vec{x}}^{R, 0}$ and notice that $\operatorname{Card}\left(V_{\vec{x}}^{D / R}\right) \leq$ $b_{0} \cdot b_{0}^{k}=b_{0}^{k+1}$ for some $k \in \omega$. If $\psi \equiv \neg \psi_{0} \operatorname{let} V_{\vec{x}}^{\bar{D} / R}:=V_{\vec{x}}^{D / R, 0}$.

Now we prove (2) and (3) by induction on the number of logical constants in $\psi(\vec{x})$. If $\psi(\vec{x})$ is atomic it is easy to check (2) and (3). Suppose $\alpha \nvdash \exists x \leq b_{0} \psi(x)$ where $\psi \in \Sigma\left(b_{0}\right)$.

We need to show that $\alpha_{\mid V} \mid \vdash \neg \exists x \leq b_{0} \psi(x)$. Conversely suppose that for some $\beta \supseteq \alpha_{\mid V}, \beta \Vdash \exists x \leq b_{0} \psi(x)$. By definition for some $a \leq b_{0}, \beta \Vdash \psi(a)$, and by induction $\beta_{\mid V_{a}^{D} \cup V_{a}^{R}} \mid \vdash \psi(a)$. Now as $\alpha_{\mid V_{a}^{D} \cup V_{a}^{R}}=\beta_{\mid V_{a}^{D} \cup V_{a}^{R}} \quad \alpha_{\mid V_{a}^{D} \cup V_{a}^{R}} \mid \vdash \exists x \leq b_{0} \psi(x)$ and then by the extension lemma $\alpha_{\mid V} \mid \vdash \exists x \leq b_{0} \psi(x)$, which is in contradiction to the assumption $\alpha \nvdash \exists x \leq b_{0} \psi(x)$.

\subsection{Some properties of the generic objects}

We have already defined $\exists^{*}$ to be the set of formulas in which all quantifiers are either existential which appear positively or are restricted to $\left[0, b_{0}\right.$ ) (Sharply bounded quantifiers). According to our plan in order to prove the main theorem we have to prove that some (any) generic $\tilde{\alpha}$ satisfies the $\exists^{*}$-LNP scheme. Let $\exists_{\text {Strict }}^{*}$ be the set of formulas $\exists \vec{x} \psi(\vec{x})$, where $\psi \in \Sigma\left(b_{0}\right)$ and where there are no restrictions on the parameters in $\psi$. First we prove that:

Lemma 4.7.1 For any generic map $\tilde{\alpha},(\mathbb{M}, \tilde{\alpha})$ satisfies the $\exists_{\text {Strict }}^{*}$-LNP scheme.

Proof: Let $\psi(z) \equiv \exists \vec{x} \psi_{1}(\vec{x}, z)$ be given. $\left(\psi_{1} \in \Sigma\left(b_{0}\right)\right)$. Let $\alpha_{0} \in \mathcal{P}_{k}$ be given such that $\alpha_{0} \Vdash \psi(a)$ for some $a \in \mathbb{M}$. Let $a_{0} \in \mathbb{M}$ be the smallest element such that for 
some $\beta \supseteq \alpha_{0}$ with $\beta \in \mathcal{P}_{k+r}$ (for suitable $r$ ), and some $\vec{x}_{0}, \quad \beta \Vdash \psi_{1}\left(\vec{x}_{0}, a_{0}\right)$. This definition makes sense because the forcing relation is definable for $\Sigma\left(b_{0}\right)$-formulas. (By lemma 4.6.4). Let $\mathcal{D}$ be the set of $\beta^{\prime} \in \mathcal{P}$ which are either incompatible to $\alpha_{0}$ or extensions of a $\beta$ with the property just mentioned above. $\mathcal{D}$ is quasi-definable and dense so there is $\alpha \in \mathcal{D} \cap \mathcal{G}$. Clearly $\alpha \Vdash \psi\left(a_{0}\right)$. All that remains is to check that if $r$ is chosen properly (lemma 4.6.4) there is no $a_{1}<a_{0}$ and $\alpha^{\prime} \supset \alpha$ with $\alpha^{\prime} \mid \vdash \psi\left(a_{1}\right)$.

\subsection{A minor problem}

Now we want to prove that for some (any) generic $\tilde{\alpha},(\mathbb{M}, \tilde{\alpha})$ actually satisfies the $\exists^{*}$-LNP scheme. It should be noticed that because of the presence of the generic object $\tilde{\alpha}$ it is not entirely clear why any $\exists^{*}$-formula should be equivalent to an $\exists_{S \text { trict }}^{*}$ formula.

Let $\psi(z)$ be a given $\exists^{*}$-formula. Let us try to follow the same strategy as in the proof of lemma 4.7.1 above. Without loss of generality we can assume that

$$
\psi(z) \equiv \exists x_{0} \forall u_{0} \leq b_{0} \exists x_{1} \forall u_{1} \leq b_{0} \ldots \exists x_{k} \theta(\vec{x}, \vec{u}, z)
$$

where $\theta \in \Sigma\left(b_{0}\right)$. Find smallest $z_{0}$ such that there are $a_{0}, a_{1,0}, a_{1,1}, \ldots, a_{1, b}$, and $a_{2,0,0}, a_{2,0,1}, \ldots, a_{2,0, b_{0}}$ and $a_{2,1,0}, a_{2,1,1}, a_{2,1,2}, \ldots, a_{2,1, b_{0}} \ldots, a_{2, b_{0}, b_{0}}, \ldots$

$$
\cdots \underbrace{a_{k, b_{0}, b_{0}, \ldots b_{0}}}_{k b_{0}^{\prime} s}
$$

and $\beta \supseteq \alpha_{0}$ in $\mathcal{P}_{k+r}$ such that for any choice $u_{0} \leq b_{0}, u_{1} \leq b_{0}, \ldots u_{k} \leq b_{0}$

$$
\beta \Vdash \theta\left(a_{0}, a_{1, u_{0}}, a_{2, u_{0}, u_{1}}, \ldots, a_{k, u_{0}, \ldots, u_{k-1}}, u_{0}, u_{1}, \ldots u_{k-1}, z_{0}\right)
$$

Again if there is any $z_{0}$ satisfying the condition, there is also a smallest such $z_{0}$. This holds for each choice of $r \in \omega$. As a minor technical problem we need to show (what might be obvious to the reader) that if $\beta$ is chosen as above, for no $\beta^{\prime} \supseteq \beta$ we can have $\beta^{\prime} \Vdash \psi\left(z_{1}\right)$ for some $z_{1}<z_{0}$. Now in general (M, $\alpha$ ) has more definable functions on $\left[0, b_{0}\right)$ than $\mathbb{M}$. This is because for each formula $\theta(x, \alpha)$ and each generic map $\tilde{\alpha}, \alpha$ could be eliminated (i.e. there was a formula $\psi(x)$ such that $\forall x \in\left[0, b_{0}\right) \theta(x, \alpha) \Leftrightarrow \psi(x)$ in the case where $\left.f_{\text {slow }}(x):=\log (x)\right)$, then induction would hold up to $b_{0} \geq \log (c)$ and therefore up to $c$, which would be a contradiction. Essentially we have to check that $\psi\left(z_{1}\right)$ can not have a sequence of witnesses which was not definable in $\mathbb{M}$. In the case where $\psi(x)$ was a $\exists_{\text {Strict }}^{*}$-formula there was no such problem because the search in $\mathbb{M}$ was only a search for standard finitely many witnesses. We have to show that there cannot be such a $z_{1}$. This is essentially done 
by showing that in the case of $\exists^{*}$-formulas for any generic $\tilde{\alpha}$ there is an $\mathbb{M}$ definable sequence of witnesses.

For $\theta \in \exists^{*}$ we make the following definition:

Definition 4.8.1 $\alpha \vdash^{D} \forall x \leq b_{0} \theta(x)$ iff $\forall x \leq b_{0} \alpha \Vdash^{D} \theta(x)$.

$\alpha \vdash^{D} \exists x \theta(x)$ iff $\exists x \alpha \Vdash^{D} \quad \theta(x)$

$\alpha \vdash^{D} \neg \neg \theta$ iff $\alpha \vdash^{D} \quad \theta$.

Notice that for $\theta \in \exists^{*}$ if $\alpha \vdash^{D} \theta$ for each extension (not necessarily a generic extension!) $\tilde{\alpha}$ of $\alpha$ then $(\mathbb{M}, \tilde{\alpha}) \models \theta$.

Observation 4.8.2 For each $\alpha_{0} \in \mathcal{P}$, each $k \in \omega$ and each $\psi \in \exists^{*}$ the set $\{\langle\alpha, x\rangle$ : $\left.\alpha \supseteq \alpha_{0} \wedge \alpha \in \mathcal{P}_{k} \wedge \alpha \vdash^{D} \psi(x)\right\}$ is definable in $\mathbb{M}$.

The problem we are concerned with at this stage is whether it is possible for given $\alpha_{0}$ and $\theta \in \Sigma\left(b_{0}\right)$ to have $\alpha \supseteq \alpha_{0}$ with $\alpha \Vdash \forall x \leq b \exists y \theta(x, y)$ but for $\underline{\text { no }} \beta \supseteq \alpha_{0}, \beta \in$ $P_{r}: \beta \vdash^{D} \forall x \leq b \exists y \theta(x, y)$ ?

Assume that there is $\alpha \supseteq \alpha_{0}$ such that $\alpha \Vdash \forall x \leq b_{0} \exists y \theta(x, y)$.

Claim: There is an extension $\alpha_{1} \supseteq \alpha$ such that $\forall x \leq b_{0} \exists y \alpha_{1} \Vdash \theta(x, y)$. Pick for each $j \leq b_{0}, \alpha^{j}$ such that $\alpha \subseteq \alpha^{0} \subseteq \alpha^{1} \subseteq \ldots \alpha^{b_{0}}=\alpha_{1}$ and such that $\alpha_{j} \Vdash \exists y \theta(j, y)$. Furthermore by lemma 4.6.4 each extension can be chosen to be of size $\leq b_{0}^{l}$ for some fixed $l \in \omega$ ( $l$ can be chosen to be the number of " $\forall x \leq b_{0}$ " quantifiers in $\theta$ ). So $\alpha_{1}$ is a $\leq b_{0}^{l+1}$ extension of $\alpha$. By lemma 4.6.4 for each $x \leq b_{0}$ and witness $y(x)$ there is a set $V_{x, y(x)}$ with cardinality $\leq b_{0}^{l}$ for some fixed standard $l$. Let $V:=\bigcup_{x \leq b_{0}} V_{x, y(x)}$. Notice that $\operatorname{Card}(V) \leq b_{0}^{l+1}$ and that $\forall x \leq b_{0} \exists y \alpha_{1, \mid V} \Vdash \psi(x, y)$. Let $\beta=\left(\alpha_{1}\right)_{\mid V}$ and notice that $\left.\beta\right|^{D} \forall x \leq b_{0} \exists y \theta(x, y)$. We have just proved the first part of the next lemma:

Lemma 4.8.3 Let $\psi(z)=\forall x \leq b_{0} \exists y \theta(x, y, z)$ where $\theta \in \Sigma\left(b_{0}\right)$. There is $r \in \omega$ such that if $\beta \supseteq \alpha_{0}$ is the $b_{0}^{r}$-extension of $\alpha_{0}$ with the smallest $z_{0}$ such that

$$
\beta \vdash^{D} \forall x \leq b_{0} \exists y \theta\left(x, y, z_{0}\right)
$$

then for any generic model (M, $\tilde{\alpha})$ with $\tilde{\alpha} \supseteq \alpha_{0} \quad(\mathbb{M}, \tilde{\alpha}) \models \psi\left(z_{0}\right) \wedge \forall z<z_{0} \neg \psi(z)$.

More generally Let $\psi(z)=\exists x_{0} \forall u_{0} \leq b_{0} \exists x_{1} \forall u_{1} \leq b_{0} \ldots \exists x_{k} \theta(\vec{u}, \vec{x}, z)$ where $\theta \in$ $\Sigma\left(b_{0}\right)$. Then there is a standard number $r$ which does not depend on the parameters in $\psi$ such that if $\beta \supseteq \alpha_{0}$ is the $b_{0}^{r}$-extension of $\alpha_{0}$ with the smallest $z_{0}$ satisfying $\left.\beta\right|^{D} \quad \psi\left(z_{0}\right)$ then for any generic model (M, $\left.\tilde{\alpha}\right)$ with $\tilde{\alpha} \supseteq \alpha_{0}$

$$
(\mathbb{M}, \tilde{\alpha}) \models \psi\left(z_{0}\right) \wedge \forall z<z_{0} \neg \psi(z) .
$$


Proof: Let $\alpha_{0}$ be given. Assume that for some $\alpha \supseteq \alpha_{0}$

$$
\alpha \Vdash \forall x_{1} \leq b_{0} \exists y_{1} \forall x_{2} \leq b_{0} \ldots \exists x_{k} \theta(\vec{x}, \vec{y})
$$

but that for no $\beta \supseteq \alpha_{0},\left.\beta \in \mathcal{P}_{r} \quad \beta\right|^{D} \forall x_{1} \leq b_{0} \exists y_{1} \ldots \theta(\vec{x}, \vec{y})$. There is $\alpha^{\prime} \supseteq \alpha$ such that $\forall x_{1} \leq b_{0} \exists y_{1} \alpha^{\prime} \Vdash \forall x_{2} \leq b_{0} \exists y_{2} \ldots \theta(\vec{x}, \vec{y})$. By induction on $k$, we can assume that we have already proved that if for some fixed $x_{1}$ and $y_{1}, \beta \supseteq \alpha, \beta \Vdash \forall x_{2} \leq$ $b_{0} \exists y_{2} \ldots \theta(\vec{x}, \vec{y}), \beta$ can be chosen to be an $b_{0}^{l+k-1}$-extension, where $l$ is the number of $\forall$-quantifiers in $\theta$. There is $\alpha^{\prime \prime} \supseteq \alpha^{\prime}$ (making $b_{0}^{2} \quad b_{0}^{k+l-2}$-extensions of $\alpha^{\prime}$ ), such that

$$
\forall x_{1} \leq b_{0} \exists y_{1} \forall x_{2} \leq b_{0} \exists y_{2} \alpha^{\prime \prime} \mid \vdash \forall x_{3} \leq b_{0} \ldots \theta(\vec{x}, \vec{y})
$$

Continuing like this, after $k$ steps we obtain $\alpha^{*} \supseteq \alpha$ such that $\alpha^{*} \vdash^{D} \psi$. We can not use $\alpha^{*}$ as $\beta$ in the lemma, because $\alpha$ (and therefore $\alpha^{*}$ ) could be too large. But by lemma 4.6.4, there are $b_{0}^{k}$ sets $V_{x_{1}, y\left(x_{1}\right), x_{2}, y\left(x_{2}\right), \ldots, x_{k}, y\left(x_{k}\right)}$ each with $\leq b_{0}^{l}$ elements where $l$ can be taken to be the number of $\forall$-quantifiers in $\theta$. Let

$$
V:=\bigcup_{x_{1} \leq b_{0}} \bigcup_{x_{2} \leq b_{0}} \ldots \bigcup_{x_{k} \leq b_{0}} V_{x_{1}, y\left(x_{1}\right), \ldots, x_{k}, y\left(x_{k}\right)} .
$$

Notice that $\operatorname{Card}(V) \leq b_{0}^{k+l}$. Let $\beta:=\alpha_{\mid V}^{*}$ and notice that $\left.\beta\right|^{D} \psi$. This contradicts the assumption.

Corollary 4.8.4 For any generic map $\tilde{\alpha} \quad(\mathbb{M}, \tilde{\alpha}) \models \exists^{*}-\mathrm{LNP}$

Proof: Let $\tilde{\alpha}$ be an arbitrary generic map. We need to show that $(\mathbb{M}, \tilde{\alpha}) \models \exists x \psi(x) \Rightarrow$ $\exists x_{0} \leq x \forall z<x_{0} \psi\left(x_{0}\right) \wedge \neg \psi(z)$. Suppose $(\mathbb{M}, \tilde{\alpha}) \models \psi(a)$ for some $a \in \mathbb{M}$ (otherwise there is nothing to prove). According to the completeness property there is $\alpha_{0} \subseteq \tilde{\alpha}$ such that $\alpha_{0} \in P$ and $\alpha_{0} \Vdash \psi(a)$. Assume $\alpha_{0} \in \mathcal{P}_{k}$. Consider $\mathcal{D} \subseteq \mathcal{P}$ defined by $\mathcal{D}:=\left\{\alpha \mid \alpha \nsupseteq \alpha_{0} \vee\left(\left.\alpha \supseteq \alpha_{0} \wedge \exists x \alpha\right|^{D} \quad \psi(x) \wedge \forall y<x\left(\forall \alpha^{\prime} \supseteq \alpha \alpha^{\prime} \nvdash^{D} \quad \psi(y)\right)\right)\right\}$. From what has already been proved it follows that $\mathcal{D}$ is well defined, dense and quasidefinable. For any generic $\mathcal{G} \subseteq \mathcal{P}$ there are $\alpha_{1} \in \mathcal{D} \cap \mathcal{G}$. By lemma 4.8.3 for any $\tilde{\beta} \supseteq \alpha$ (in particular $\tilde{\alpha})(\mathbb{M}, \tilde{\beta}) \models \exists x \psi(x) \Rightarrow \exists x_{0} \leq x \forall z<x_{0} \psi\left(x_{0}\right) \wedge \neg \psi(z)$. As $\alpha_{1} \subseteq \tilde{\alpha}$ we are done.

\subsection{Proof of the first finitisation principle}

The previous section has given us a "generic model" ( $\mathbb{M}, \tilde{\alpha})$ which satisfies the $\exists *$-LNP scheme. Clearly $\tilde{\alpha}$ induces a miniaturised version of $S$ on $[0, n)$. Constants, relations and functions on $S$ correspond to constants, relations and functions on $[0, n)$. Now I prove the important fact that all the miniaturised relations etc. are $\exists \cap \forall$-definable in $(\mathbb{M}, \tilde{\alpha})$. 
For each relation $R \subseteq S^{r}$ (with quantifier-free definition in $\mathbb{M}$ ), we define the corresponding miniaturised relation $R_{\text {mini }}$ "existentially" by:

$$
\left\{\left(x_{1}, x_{2}, \ldots, x_{r}\right) \mid \exists z_{1}, z_{2}, \ldots, z_{r} \wedge_{i=0}^{i=r} \tilde{\alpha}\left(x_{i}, z_{i}\right) \wedge R\left(z_{1}, z_{2}, \ldots, z_{r}\right)\right\} .
$$

Notice that $R_{\text {mini }}$ also has a "universal" definition:

$$
\left\{\left(x_{1}, x_{2}, \ldots, x_{r}\right) \mid \forall z_{1}, z_{2}, \ldots, z_{r} \wedge_{i=0}^{i=r} \quad \tilde{\alpha}\left(x_{i}, z_{i}\right) \Rightarrow R\left(z_{1}, z_{2}, \ldots, z_{r}\right)\right\}
$$

Add the miniaturised relations to the language. Consider the sub-language $L_{\text {mini }}$ which contains $L$ and names for the miniaturised constants, relations and functions.

Lemma 4.9.1 If $\psi(x) \in L_{\text {mini }}$ is a $\Sigma_{1}^{b}$-formula with all quantifiers restricted to $[0, c)$, there is $a \exists^{*}$-formula $\psi^{*}(x)$ such that:

$$
\{x \leq c:(\mathbb{M}, \tilde{\alpha}) \models \psi(x)\}=\left\{x \leq c:(\mathbb{M}, \tilde{\alpha}) \models \psi^{*}(x)\right\} .
$$

Proof: Let $\psi(x) \in L_{\text {mini }}$ be a given $\Sigma_{1}^{b}$ property. Replace each appearance of a "miniaturised" relation $R_{\text {mini }}$ by either the $\exists$-definition or the $\forall$-definition according to whether the $R_{\text {mini }}$ appears positively or negatively in $\psi(x)$. Notice that this gives an $\exists^{*}$-formula $\psi^{*}(x)$ which satisfies the lemma.

Now we are ready to construct a model of $T_{2}^{1}(\alpha)$ in which there is a bounded sub-structure $S_{\text {mini }}$ on $[0, n)$ where $\eta(n)$ such that $S_{\text {mini }} \models \theta_{<n}(\vec{R})$. We have already got a countable model $\mathbb{M}$ of true arithmetic in which a possibly unbounded model of $\theta(\vec{R})$ is coded. We have $n, b_{0}, c \in \mathbb{M}$ as above. Let $\tilde{\alpha}$ be an arbitrary generic map, as described earlier. Let

$$
\mathbb{M}_{\tilde{\alpha}}^{*}=\left\{x \leq c: \exists k \in \omega \quad x<f_{\text {slow }}^{(k)}(n)\right\} .
$$

Let $\hat{R}$ be the set of all $L_{m i n}$-definable relations on $[0, n)$. It follows from what has already been proved that $(\mathbb{M}, \hat{R}) \models \Sigma_{1}^{b}$-IND scheme, hence because of corollary 3.4.5 there is an expansion of $\hat{R}$ to $\hat{S} \supseteq \hat{R}$ such that $(\mathbb{M}, \hat{S}) \models T^{1}(\alpha)$. As second order existence statements are absolute with respect to expansions, we have proved the main theorem in the $T^{1}(\alpha)$-case.

Corollary 4.9.2 Let $S$ be a countable mathematical structure which can be encoded in a non-standard model $\mathbb{M}$ which satisfies true arithmetic. There is a model of $T_{2}^{1}(\alpha)$ in which $S$ appears as a bounded set.

Notice that our results hold in the special case where $T^{1}(\alpha)$ is the second order theory which consists of

1) Induction for existential formulas.

2) The $\forall \cap \exists$-comprehension axiom scheme. 


\section{$5 \quad$ Separating $T_{2}^{1}(\alpha)$ and $S_{2}^{2}(\alpha)$}

In the process as a by-product we have obtained a new proof of the separation of the theories $T_{2}^{1}(\alpha)$ and $S_{2}^{2}(\alpha)$.

Theorem 5.0.3 For any generic map $\tilde{\alpha}\left(\mathbb{M}_{\tilde{\alpha}}^{*}, \tilde{S}_{\tilde{\alpha}}\right) \models T_{2}^{1}(\alpha)$.

If $S$ defines two disjoint infinite sets, then for any generic map $\tilde{\alpha}\left(\mathbb{M}_{\tilde{\alpha}}^{*}, \tilde{S}_{\tilde{\alpha}}\right) \forall$ $S_{2}^{2}(\alpha)$

Proof: The first part of the theorem has already been proven. It follows from the examples below that the second part holds at least for some constructions (i.e. for some $S)$. To prove the second part let $U(x)$ be a new unary predicate symbol which holds exactly in one component of $S$. Let $U_{\operatorname{mini}}$ denote the corresponding predicate in $L_{\text {mini }}$. Put $f_{\text {slow }}:=|x|$ and $f_{\text {fast }}:=x^{|x|}$. Consider the formula

$$
A(z):=\exists u_{1}<u_{2} \leq n u_{2}-u_{1} \geq b^{z} \forall x \in\left[u_{1}, u_{2}\right) U_{\operatorname{mini}}(x) .
$$

Clearly $A(k)$ is valid for all standard $k$ and hence, by overspill, $A(z)$ holds for some non-standard $z$ (and $z \leq|n|$ ). But this is a contradiction as for any given interval of length $<b^{\omega}$ the set of $\alpha \in \mathcal{P}$ such that $\alpha$ maps an element of $S \backslash U$ into it, is dense and quasi-definable.

According to [5] $\Sigma_{2}^{b}$-PIND is sufficient to ensure the validity of $\Sigma_{2}^{b}$-LMAX principle which in turn implies the validity of the $\Sigma_{2}^{b}$-overspill just used.

Notice that if $S$ is an infinite (co-countable) set with no additional structure then $\left(\mathbb{M}_{\tilde{\alpha}}^{*}, \tilde{S}_{\tilde{\alpha}}\right) \models S_{2}(\alpha)$. This shows that the second part of the theorem becomes false if there is no condition on $S$.

Corollary 5.0.4 $T_{2}^{1}(\alpha) \neq S_{2}^{2}(\alpha)$.

This is a new proof of the result which was first proved in [8].

\section{Some examples}

Example 6.0.5 Fix $p \geq 2$. There is an infinite model $A$ where "R defines a partition of $A$ into disjoint $p$-subsets". Let $\eta(n) \equiv$ " $n$ is not divisible by $p$ '.

According to the first principle $T^{1}(\alpha) \forall \operatorname{Count}(p)$.

In [2] and [16] it is shown that this holds for much stronger theories. 
Example 6.0.6 According to the first principle:

$T^{1}(\alpha) \forall$ "every linear ordering $R$ (of a finite set) has an isolated point". $T^{1}(\alpha) \forall$ "every linear ordering $R$ (of a finite set) is discrete".

$T^{1}(\alpha) \forall$ "every linear ordering $R$ (of a finite set) is a well ordering".

By the results below in all cases $T^{1}(\alpha)$ can be replaced by $S_{2}^{2}(\alpha)$.

Notice that example 6.0.6 shows (using $T_{2}^{1}(\alpha) \equiv$ WOA, Proposition 8.0.9) that the well ordering axiom for arbitrary linear orderings (WOA*) $R$ does not follow from WOA.

\section{Another principle}

The second finitisation principle says that for any given $r \in \mathbb{N}$ if some second order existential relational property $\tilde{P} \equiv P(\vec{R}, X)$ is only witnessed by infinite sets (in the real universe), there are models of $T_{2}^{1}(\alpha)$ in which there is an $n$ and relations $\vec{R} \subseteq[0, n)$ such that no subset $X \subseteq[0, n)$ with size $\leq \log _{2}^{r}(n)$ witness $P(\vec{R}, X)$.

Theorem 7.0.7 (Finitisation principle) Let $\theta \equiv \exists X \psi(\vec{R}, X)$ be a second order existential formula where $\psi$ is a first order formula in the language $L(\vec{R}, X,=)$. Let $k \in \mathbb{N}$ be a given natural number. In general (1) implies (D):

(1) There is a countable model $S$ of the language $L(\vec{R},=)$, such that for no finite set $X \subseteq S, S \models \psi(\vec{R}, X)$.

(2) $\quad T^{1}(\alpha)+{ }^{*} \exists n \forall X[0, n) \operatorname{Card}(X) \leq(\log (n))^{k} \Rightarrow \neg \psi_{<n}(\vec{R}, X)$ " has a model.

This principle states that if a second order existential property has (in the real universe) a countable model where the existential quantifier is not finitely witnessed then it is consistent with $T_{2}^{1}(\alpha)$ (and by the results below $S_{2}^{2}(\alpha)$ ) that there is a finite model where the existential quantifiers is not "polylog"-witnessed.

Example 7.0.8 In the real world there is a binary tree with no finite branch. By the second finitisation principle $T_{2}^{1}$ is consistent with the existence of a tree $T \subseteq[0, n)$, which has no branch (coded by a number) of length $\leq \log _{2}^{k}(n)$ ".

In the real word there is a vector space over $Z_{2}$ with no finite basis, so by the second principle there exists a model of $T_{2}^{1}(\alpha)$ in which there exists a vector space $V \subseteq[0, n)$ with no basic (coded by a number) of size $\leq \log _{2}^{k}(n)$ ".

Proof: Proved by a construction very similar to the proof of the first finitisation principle. Pick $S$ according to (1). Choose $S$ as a countable model in the standard 
model on say the even numbers. Extend the standard model to an elementary equivalent countable non-standard model. Pick non-standard numbers $b_{0}<n<c$ as above. Consider the same set of forcing conditions $(\mathcal{P}, \subseteq)$ as above. The forcing conditions ensure that each generic $\tilde{\alpha}$ maps small (i.e. $\leq b_{0}^{k}$ points) $\mathbb{M}$-definable sets to "small" M-definable sets. The map $\tilde{\alpha}$ induces a miniaturised structure $S_{\operatorname{mini}}$ with underlying set $[0, n)$. For each $\mathbb{M}$-definable subset $B \subseteq[0, n), A:=\tilde{\alpha}^{-1}(B)$ can not witness $\psi(\vec{R}, A)$ because the set $A$ has cardinality $\leq b_{0}^{k}$ which is finite in the model $\mathbb{M}$. As in the proof of the first principle without any complications we construct a model $T_{2}^{1}(\alpha)$ where $\psi_{<n}(\vec{R}, X)$ is not witnessed by any set $A$ of size $\leq b_{0}^{\omega}$.

\section{The well ordering axiom in $S_{2}^{1}$.}

The well ordering axiom (WOA) is the principle:

WOA

$$
\forall X(X \neq \emptyset \Rightarrow \exists y(y \in X \wedge \forall z<y \quad z \notin X))
$$

WOA says that " $<$ " well orders any set $X$, and should not be confused with the stronger principle WOA* stating that any linear ordering of a bounded set is a well ordering.

Proposition 8.0.9 (Suggested by A.J.Wilkie) WOA is equivalent to $T_{2}^{1}(\alpha)$ in models of $S_{2}^{1}(\alpha)$

Proof: $T_{2}^{1}(\alpha) \Rightarrow$ WOA: To reach a contradiction let $(\mathbb{M}, \hat{S}) \models T_{2}^{1}(\alpha)+\neg$ WOA. There is $R \in \hat{S}$ such that $R \neq \emptyset$ and such that $(\mathbb{M}, \hat{S}) \models \forall y(y \in R \Rightarrow \exists z<y z \in R)$. As $R \neq \emptyset$ there is $u_{0} \in R$ such that:

$$
(\mathbb{M}, \hat{S}) \models \forall y \leq u_{0}(y \in R \Rightarrow \exists z<y \in R)
$$

Let $Y=\left\{x \in \mathbb{M}: \exists z \in R \quad z<x \wedge x \leq u_{0}\right\} \subseteq \mathbb{M}$. Notice that $Y$ is $\Sigma_{1}^{b}$-definable in $\mathbb{M}$ with set parameter $R$ ( $Y$ is not required to belong to $\tilde{S})$. As $\Sigma_{1}^{b}$-LNP $\Leftrightarrow \Sigma_{1}^{b}$ IND, and as $Y$ is non-empty, there is a minimal $x_{0} \in Y$. By definition of $Y$ there is $z<x_{0}$ such that $z \in R$. By $(*)$ this contradicts the minimality of $x_{0}$.

WOA $\Rightarrow T_{2}^{1}(\alpha)$ : Let $(\mathbb{M}, \hat{S}) \models S_{2}^{1}(\alpha)+$ WOA be an arbitrary model. Let $\psi \in$ $\Sigma_{0}^{b} \quad\left(=\Pi_{0}^{b}\right)$ be an arbitrary formula with possible set variables $\vec{Z} \in \hat{S}$. It is enough to prove that the $\Sigma_{1}^{b}$-definable set $Y:=\{x \in \mathbb{M}: \exists z \leq t(x) \psi(x, z, \vec{Z})\} \subseteq \mathbb{M}$ has a smallest element if it is not empty. Pick $u_{0} \in Y$. To this end consider

$$
\phi(w): \equiv \exists w_{1} \leq u_{0} \exists w_{2} \leq u_{0} w=w_{1} \cdot u_{0}+w_{2} \wedge \psi\left(w_{1}, w_{2} ; \vec{Z}\right) .
$$


As $\phi \in \Delta_{1}^{b} \quad R_{b, u_{0}}: \equiv\{w \leq b \mid \phi(w)\} \in \hat{S}$ for any $b, u \in M$. Choose $b$ such that for each pair $(x, z)$ with $z \leq t(x)$ and $x \leq u_{0},(x, z) \leq b$. According to WOA $R_{b, u_{0}}$ has a smallest element $w_{0}$. Let $y_{0}$ be the unique element with $y_{0} \cdot u_{0} \leq w_{0}<\left(y_{0}+1\right) \cdot u_{0}$, and notice that $M \models y_{0}=\min (Y)$. We used the fact that strict- $\Sigma_{1}^{b}$-LNP is equivalent to $\Sigma_{1}^{b}$-IND over $S_{2}^{1}(\alpha)$. So to prove Proposition 8.0.9 all that remains is to prove this, and the equivalence of $\Sigma_{1}^{b}$-IND and strict- $\Sigma_{1}^{b}$-LNP over $S_{2}^{1}(\alpha)$. Both these facts follow from [4],[7].

\subsection{A finitisation principle for $S_{2}^{1}(\alpha)$}

As already proved $T_{2}^{1}(\alpha)$ is equivalent to the well ordering axiom WOA over the theory $S_{2}^{1}(\alpha)$. In this section I show that $S_{2}^{1}(\alpha)$ does not prove this axiom, and thus as a corollary we get a new proof of the separation result $S_{2}^{1}(\alpha) \neq T_{2}^{1}(\alpha)$. Parts of the argument are very similar to the proof of the two previous principles so I only emphasise the new ideas in the construction.

Theorem 8.1.1 (Finitisation principle for $S_{2}^{1}(\alpha)$ ) Let $\psi(\prec, R)$ be a sentence in the first order language $L(\prec, R,=)$. Suppose that $\psi(\prec, R)$ has an infinite model $S$ where $\prec$ defines a total linear ordering. Then $\exists n \exists U \subseteq[1, n] \psi_{U}(<, R)$ holds in some models of $S_{2}^{1}(\alpha)$.

Proof: I put the emphasis on the new ideas. Let $\left(\mathbb{N}, S_{\mathbb{N}}, R_{\mathbb{N}}\right)$ be an expansion of the standard model $\mathbb{N}$ to an infinite model of $S$, where $R \subseteq S^{r}$, and where $\psi(\prec, R)$ holds. Let ( $\mathbb{M}, S_{\text {large }}, R_{\text {large }}$ ) be an elementary equivalent (in a language expanded by relation symbols for $R_{\text {large }}$ and $S_{\text {large }}$ ) non-standard model. Let $n \in \mathbb{M}$ be a nonstandard number, and let $I^{*} \subseteq \mathbb{M}$ be the initial sequent defined by

$$
I^{*}:=\{m \in \mathbb{M}: \exists t \text { term } m<t(n)\} .
$$

A $\Sigma_{1}^{b}\left(I^{*}\right)$-formula $\theta$, is a $\Sigma_{1}^{b}$-formula where all parameters belong to $I^{*}$. Notice that all quantifiers in such a $\theta$ also are restricted to $I^{*}$. Choose $b_{0}$ such that $I^{*}<2^{b_{0}}$, and such that $b_{0}^{\omega}<n$. Notice that sharply bounded quantifiers in a $\Sigma_{1}^{b}\left(I^{*}\right)$-formula are bound by $b_{0}$.

We want to construct $U \subseteq[1, n]$ and $R \subseteq U^{r}$ such that $\psi_{U}(<, R)$ is forced true, and such that the polynomial induction scheme

$$
\left(\theta(0) \wedge \forall x\left(\theta\left(\left\lfloor\frac{x}{2}\right\rfloor\right) \Rightarrow \theta(x)\right)\right) \Rightarrow \forall x \theta(x)
$$

is forced true for all $\Sigma_{1}^{b}\left(I^{*}\right)$-formulas $\theta$. Let $\left\langle\theta_{1}(x), a_{1}>,<\theta_{2}, a_{2}>, \ldots\right.$ be an enumeration of the countably many pairs $\left\langle\theta_{j}(x), a_{j}\right\rangle$ where $\theta_{j}(x)$ is a $\Sigma_{1}^{b}\left(I^{*}\right)$ formula with one free variable (namely $x$ ), and where $a_{j} \in I^{*}$. 
Let $U_{0}=W_{0}=R_{0}=\emptyset$. Choose $d_{0}<e_{0} \in \mathbb{M}$ with $e_{0}-d_{0}>b_{0}^{\omega}$, and $e_{0}<\frac{n}{b_{0}}$. Let $f_{0}=\emptyset$.

In general after stage $j$ we have constructed $U_{j}, W_{j} \subseteq[1, n]$ and $R_{j} \subseteq U_{j}^{r}$, such that $U_{j} \cap W_{j}=\emptyset$. The map $f_{j}$ maps $U_{j}$ bijectively onto a subset of $S_{\text {large }}$, such that $<u_{1}, . ., u_{r}>\in R_{j}$ precisely when $R_{\text {large }}\left(f\left(u_{1}\right), \ldots, f\left(u_{r}\right)\right)$. Furthermore, we have $\left|U_{j}\right| \leq j \cdot b_{0},\left|W_{j}\right| \leq j \cdot b_{0}$, and $\left[d_{j}, e_{j}\right]$ such that $e_{j}-d_{j}>b_{0}^{\omega}$. The points in $U_{j}$ are very sparsely distributed in the sense that $\forall u_{1} \neq u_{2} \in U_{j}\left|u_{2}-u_{1}\right| \geq e_{j}$.

Now consider $\left\langle\theta_{j+1}(x), a_{j+1}>\right.$. Our aim is to force

$$
\theta_{j+1}(0) \wedge \forall x\left(\theta_{j+1}\left(\left\lfloor\frac{x}{2}\right\rfloor\right) \Rightarrow \theta_{j+1}(x)\right) \Rightarrow \theta_{j+1}\left(a_{j+1}\right)
$$

true. Let $a_{j+1}^{1}:=a_{j+1}, \ldots, a_{j+1}^{r+1}:=\left\lfloor\frac{a_{j+1}^{r}}{2}\right\rfloor, \ldots$ for $r \leq\left\lceil\log \left(a_{j+1}\right)\right\rceil$. Now for each $u \in\left[d_{j}, e_{j}\right]$ define the number $l(u) \in \mathbb{M}$ as the largest number $\left(\leq \log \left(a_{j+1}\right)\right)$ such that there are extensions $U_{j+1}^{\prime} \supseteq U_{j}, W_{j+1}^{\prime} \supseteq W_{j}$, and $R_{j+1}^{\prime} \supseteq R_{j}$, such that

(1) $\left(U_{j+1}^{\prime}, R_{j+1}^{\prime}\right)$ are isomorphic by an extension $f_{j+1}^{\prime} \supseteq f_{j}$ to a subset of $S_{\text {large. }}$.

(2) The elements in $U_{j+1}^{\prime}$ has pairwise distance $\geq u$

(3) $\left|U_{j+1}^{\prime}\right| \leq(j+1) \cdot b_{0}$

(4) $\left|W_{j+1}^{\prime}\right| \leq(j+1) \cdot b_{0}$.

(5) At least $l(u)$ of the $\Sigma_{1}^{b}\left(I^{*}\right)$-formulas $\theta\left(a_{j+1}^{1}\right), \ldots, \theta\left(a_{j+1}^{\left\lceil\log \left(a_{j+1}\right)\right\rceil}\right)$ are forced true.

Now because $l$ takes less than $b_{0}$ values, there must be an interval $\left[d_{j+1}, e_{j+1}\right] \subseteq\left[d_{j}, e_{j}\right]$ such that $l$ is constant on this interval, and such that $e_{j+1}-d_{j+1}>b_{0}^{\omega}$. Choose such an interval, and extend $U_{j}, W_{j}$ and $R_{j}$ by letting $U_{j+1}:=U_{j+1}^{\prime}, W_{j+1}:=W_{j+1}^{\prime}, R_{j+1}:=$ $R_{j+1}^{\prime}$, and $f_{j+1}:=f_{j+1}^{\prime}$ where these extensions correspond to the case where $u=e_{j+1}$. Proceed with the construction in this way:

Let $U:=\cup_{j \in \omega} U_{j}$ and let $R=\cup_{j \in \omega} R_{j}$ and notice $R \subseteq U^{r}$. It is not hard to show that $f=\cup_{j \in \omega} f_{j}$ defines a bijection from the miniaturised model $U$ on $[1, n]$ to the large model $S_{\text {large. }}$ This ensures that $\psi_{U}(<, R)$ holds in the generic model.

The $\Sigma_{1}^{b}\left(I^{*}\right)$ polynomial induction schema is forced true. To see this suppose contrarily that for some $k \in \omega$ in the generic model we have

$$
\theta_{k}(0) \wedge \forall x\left(\theta_{k}\left(\left\lfloor\frac{x}{2}\right\rfloor\right) \Rightarrow \theta_{k}(x)\right) \wedge \neg \theta_{k}\left(a_{k}\right) .
$$

In stage $k$ in the construction we have forced a maximum number of the $\Sigma_{1}^{b}\left(I^{*}\right)$ formulas $\theta_{k}\left(a_{k}^{1}\right), \theta_{k}\left(a_{k}^{2}\right), . ., \theta_{k}\left(a_{k}^{j}\right), . ., \theta_{k}(0)$ true. As obviously $\theta_{k}\left(a_{k}\right)$ cannot have been forced true, there must be $j<\left\lceil\log \left(a_{k}\right)\right\rceil$ such that $\theta_{k}\left(a_{k}^{j+1}\right)$ is already forced true (after stage $k$ ) while $\theta_{k}\left(a_{k}^{j}\right)$ is not forced true at this stage (here we are using that the search procedure, for fixed $u \in\left[d_{k}, e_{k}\right]$, is $\mathbb{M}$-definable). Could it be that $\theta_{k}\left(a_{k}^{j}\right)$ get forced true at a later stage $k^{\prime}>k$ ? No, because if this were the case for some $u \in\left[d_{k}, e_{k}\right], l$ would take a larger value than $l\left(e_{k}\right)$, which would be a contradiction. 
Corollary 8.1.2 There are models of $S_{2}^{1}(\alpha)$ in which WOA fails.

Proof: Let $\psi(\prec):=\forall x \exists y y \prec x$. This sentence holds in an infinite model (in the real world), so by theorem 8.1.1 the sentence $\forall x \in U \exists y \in U y<x$ holds in some models of $S_{2}^{1}(\alpha)$.

This gives a new proof of the separation first proved in [10]

Corollary 8.1.3 $S_{2}^{1}(\alpha) \neq T_{2}^{1}(\alpha)$.

\section{Lifting the finitisation principles}

Now I show that both finitisation principles for $T_{2}^{1}(\alpha)$ hold for the theory $S_{2}^{2}(\alpha)$ but both fail to hold for $T_{2}^{2}(\alpha)$. This, in particular separates the theories $S_{2}^{2}(\alpha)$ and $T_{2}^{2}(\alpha)$. In this I rely heavily on a proof sketch ed by J.Krajicek (personal communication). The proof relies again strongly on S.Buss' result [5], that $S_{2}^{i+1}(\alpha)$ is $\forall \Sigma_{i+1}^{b}$-conservative over $T_{2}^{i}(\alpha)$.

Theorem 9.0.4 The first finitisation principle holds if $T^{1}(\alpha)$ is replaced by $S_{2}^{2}(\alpha)$

Proof: To get a contradiction assume that $\theta(R) \equiv \forall x_{1} \exists y_{1} \ldots \forall x_{k} \exists y_{k} \tilde{\theta}(\vec{x}, \vec{y} ; R)(\tilde{\theta}$ is quantifier free) does have an infinite model, but $S_{2}^{2}(\alpha) \vdash \forall n(\eta(n) \Rightarrow \forall R<$ $\left.n \neg \theta_{<n}(R)\right)$. Consider the skolemisation

$$
\tilde{\theta}\left(x_{1}, F_{1}\left(x_{1}\right), x_{2}, F_{2}\left(x_{1}, x_{2}\right), \ldots, x_{k}, F_{k}\left(x_{1}, x_{2}, \ldots, x_{k}\right) ; R\right)
$$

of $\theta(R)$. By the first finitisation principle $T_{2}^{1}(\alpha)+\exists n\left(\eta(n) \wedge \exists R<n \exists F_{1}, . . \exists F_{k}<n\right.$ $\left.\forall \vec{x} \leq n \tilde{\theta}\left(x_{1}, F_{1}\left(x_{1}\right) \ldots ; R\right)\right)$ is consistent.

Add an extra constant $\bar{n}$, extra function symbols $\bar{F}_{1}, \bar{F}_{2}, . ., \bar{F}_{k}$ and an extra relational symbol $\bar{R}$ to the language. Call this language $\tilde{L}_{2}$. The first order theory $T_{2}^{1}\left(\bar{n}, \bar{F}_{1}, \ldots, \bar{F}_{k}, \bar{R}\right)+\forall x \leq \bar{n} \tilde{\theta}\left(x_{1}, \bar{F}_{1}\left(x_{1}\right), \ldots, \bar{F}_{k}(\vec{x}) ; \bar{R}\right)$ is consistent.

Now by theorem 3.5.3 $S_{2}^{2}\left(\bar{n}, \bar{F}_{1}, \ldots ., \bar{F}_{k}, \bar{R}\right)+\forall x \leq \bar{n} \tilde{\theta}\left(x_{1}, \bar{F}_{1}\left(x_{1}\right), \ldots, \bar{F}_{k}(\vec{x}) ; \bar{R}\right)$ is consistent. As a skolemisation of $\theta$ implies $\theta$ (even in pure predicate logic) this contradicts our initial assumption about $S_{2}^{2}(\alpha)$.

This give us the following:

Corollary 9.0.5 Let $\theta(\vec{R})$ be any first order property in some relational language $\tilde{L}:=L(\vec{R})$. Then the following are equivalent:

$$
\text { Predicate logic } \vdash \theta(\vec{R})
$$




$$
T^{1}(\alpha) \vdash \forall n \theta_{<n}(\vec{R})
$$

$$
S_{2}^{2}(\alpha) \vdash \forall n \theta_{<n}(\vec{R})
$$

Proof: Let $T$ be $T^{1}(\alpha)$ or $S_{2}^{2}(\alpha)$. Assume that $\theta(\vec{R})$. Assume that $T$ does not prove $\forall n \theta_{<n}(\vec{R})$. There exists a non-standard model $\mathbb{M}^{*}$ of $T$ in which $\neg \theta_{<n}(\vec{R})$ holds in some initial segment $[0, n)$, so $([0, n), \vec{R}) \models \neg \theta(\vec{R})$. But then by the completeness theorem $\theta(\vec{R})$ is not provable in predicate logic.

Contrarily if predicate logic does not prove $\theta(\vec{R})$ there is a model $S$ of $\neg \theta(\vec{R})$. If $S$ is finite certainly $\forall n \theta_{<n}(\vec{R})$ does not have a $T$-proof (because $T$ is consistent). If $S$ is infinite, by the finitisation principle $\exists n \neg \theta_{<n}(\vec{R})$ is consistent with $T$.

\section{Separating $S_{2}^{2}(\alpha)$ and $T_{2}^{2}(\alpha)$}

Theorem 10.0.6 The statement "Every linear ordering of an interval has a smallest

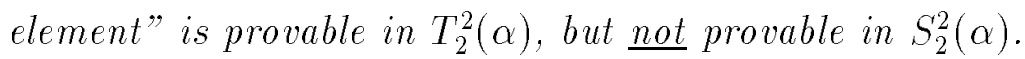

The statement "There is no dense linear ordering of an interval of length $\geq 2$ " is provable in $T_{2}^{2}(\alpha)$ but not provable in $S_{2}^{2}(\alpha)$.

Proof: The second part of the claim follows from the finitisation principle for $S_{2}^{2}(\alpha)$. To prove the first part let $(\mathbb{M}, \hat{S}) \models T_{2}^{2}(\alpha)$ be an arbitrary model. Assume that $R$ is an $\mathbb{M}$-definable linear ordering of an interval $[0, n)$ for some $n \in M$. Let

$$
Y=\left\{b \in[0, n): \exists a_{0} \leq b \forall x \leq b x R a_{0} \Rightarrow x=a_{0}\right\} .
$$

Notice that $Y$ is $\Sigma_{2}^{b}$-definable and that $0 \in Y$ and $b \in Y \Rightarrow b+1 \in Y$ for $b<n$. As $(\mathbb{M}, \hat{S}) \models \Sigma_{2}^{b}-$ IND, $(n-1) \in Y$. By definition of $Y, R$ has a smallest element. The second part follows from the first part.

This gives us a new proof of

Corollary 10.0.7 $S_{2}^{2}(\alpha) \neq T_{2}^{2}(\alpha)$.

The following were proved in [10]. Above we have obtained a new proof of the second part of the theorem.

Theorem 10.0.8 The weak pigeon-hole principle (WPHP) is provable in $T_{2}^{2}(\alpha)$, but not provable in $S_{2}^{2}(\alpha)$.

Proof: The second part of the theorem has already been proved. The first part follows from the fact that $S_{2}^{3}(\alpha)$ is $\forall \Sigma_{3}^{b}$-conservative over $T_{2}^{2}(\alpha)$, that WPHP is a $\forall \Sigma_{2}^{b}$-formula and from the following sub claim: 
Lemma 10.0.9 WPHP is provable in $S_{2}^{3}(\alpha)$.

Proof: A careful analysis of the $S_{2}$-proof in [12] (see also [8]) of WPHP shows that the proof is actually a $S_{2}^{3}(R)$-proof.

\section{Lifting the second principle}

For the sake of completeness let me sketch how the second finitisation principle can be lifted to $S_{2}^{2}(\alpha)$.

Theorem 11.0.10 Let $\theta \equiv \exists X \psi(\vec{R}, X)$ be a second order existential formula where $\psi$ can be any first order formula in the relational language $L(\vec{R},=)$. Let $k \in \mathbb{N}$ be a given natural number. In general (1) implies (D):

(1) There is a countable model $S$ of the language $L(\vec{R},=)$, such that for no finite set $X \subseteq S, S \models \theta(\vec{R}, X)$.

$$
S_{2}^{2}(\alpha)+{ } \exists n \forall X \subseteq[0, n) \operatorname{Card}(X) \leq(\log (n))^{k} \rightarrow \neg \theta_{<n}(\vec{R}, X) " \text { has a model. }
$$

Proof:(sketch) Essentially we lift the result as we did for the first finitisation principle. Suppose that $S_{2}^{2}(\alpha) \vdash \forall n \forall \vec{R} \subseteq[0, n) \exists b \psi_{<n}(\vec{R}$, Seq $(b))$ where $\psi_{<n} \equiv \forall x_{1}<n \exists x_{2}<$ $n \ldots \exists x_{2 r}<n \tilde{\theta}(\vec{x}, n, \vec{R}, \operatorname{Seq}(b))$ with $\tilde{\theta}$ quantifier free.

We have already proved the theorem in the $T_{2}^{1}(\alpha)$-case, so

$$
T_{2}^{1}(\alpha)+\forall \vec{x}<n \forall b \neg \tilde{\theta}\left(x_{1}, \bar{F}_{1}\left(x_{1}\right), x_{3}, \ldots, \bar{F}_{r}, n, \vec{R}, \operatorname{Seq}(b)\right)
$$

is consistent, where the language is extended by extra relation symbols $\bar{n}, \vec{R}, \bar{F}_{1}, \ldots, \bar{F}_{r}$. By S.Buss' conservation result, (theorem 3.5.3), which also works in this case where we have added extra function symbols to the language

$$
S_{2}^{2}(\alpha)+\forall \vec{x}<\bar{n} \forall b \neg \tilde{\theta}\left(x_{1}, \bar{F}_{1}\left(x_{1}\right), \ldots, \bar{n}, \vec{R}, \operatorname{Seq}(b)\right)
$$

is consistent in this extended language. Clearly this contradicts our initial assumption.

Notice the following phenomenon:

Corollary 11.0.11 If

$$
\exists X \subseteq[0, n) \operatorname{Card}(X) \leq(\log (n))^{k_{1}} \wedge \theta_{<n}(\vec{R}, X)
$$

holds in the real world for some constant $k_{1}$ but does not hold for some other constant $k_{2}<k_{1}, S_{2}^{2}(\alpha)$ does not prove the formula for any constant $k$. 


\section{Some applications}

By use of the second finitisation principle we get the following theorem. The last case (3) shows that the theorem is not entirely a curiosity.

Theorem 12.0.12 If $k$ is a natural number, then $S_{2}^{2}(\alpha)$ does not prove any of the following statements:

(1) "Every vector space $V \subseteq[0, n)$ over $Z_{2}$ has a basis of size $\leq \log ^{k}(n)$ ".

(2) "Every binary tree $T \subseteq[0, n)$ has a branch of length $\leq \log ^{k}(n)$ ".

(3) "Every total irreflexively oriented graph on $[0, n)$ has a dominating set of size $\leq \log ^{k}(n) "$.

On the other hand $T_{2}^{2}(\alpha)$ proves (1)-(D).

At present, it is not known whether $T_{2}^{2}(\alpha) \vdash(3)$. A proof of even $S_{2}(\alpha) \vdash(3)$ would have interesting consequences. (See $[13]$ and $[10]$ for more details).

Proof: The first part of the theorem follows from the second finitisation principle (in case (1) from a slightly modified version of the principle) and the facts:

There is an (infinite) vector space with no finite basic.

There is an (infinite) binary tree with no finite branch.

There is an (infinite) irreflexively oriented graph with no finite dominating set.

To prove the last part of the theorem, assume $V$ is a vector space on $[0, n)$ with no coded basic. Pick independent vectors $v_{1}, v_{2}, \ldots, v_{\lfloor\log (2 n)\rfloor+1}$ and get a $\Delta_{1}^{b}$-definable injective map from $[0,2 n)$ to $[0, n)$. This violates WPHP which are provable in $T_{2}^{2}(\alpha)$ by [4] (or [8]).

Finally $T_{2}^{2}(\alpha)$ proves that every tree $T \subseteq[0, n)$ has a coded branch. Let $T^{*}:=$ $\{x \in T: \exists b b$ codes a path of length $\leq\lfloor\log (n)\rfloor+1$ from the root to $x\}$. Notice that $T^{*}$ also have the $\Pi_{1}^{b}$-definition: $T^{*}:=\{x \in T: \forall b b$ codes a path from the root to $x$ and the length of the path is $\leq\lfloor\log (n)\rfloor+1\}$. Define a $\Delta_{1}^{b}$-relation by $F(x)=y$ iff $\forall b b$ codes a path $x_{1}, x_{2}, \ldots, x_{r}$ from the root to $x$, and bit $(y, j)=1 \leftrightarrow x_{j}$ is a right son. Again we get a contradiction to the WPHP in [11].

\section{References}

[1] M.Ajtai; On the complexity of the pigeonhole principle. $29^{t h}$ Annual symp. on Found. Comp.Sci.(1988),pp 340-355.

[2] M.Ajtai; Parity and the pigeon-hole principle, in Feasible Mathematics Birkhauser, (1990), pp 1-24. 
[3] A.Berrarducci, B.Intrigila; Combinatorial principles in elementary number theory, Annals of Pure and Applied Logic 55 (1991) pp 35-50.

[4] S.Buss; Bounded Arithmetic. Ph.D. dissertation, Princeton University, (1985). As book, Bibliopolis, Napoli (1986).

[5] S.Buss; Axiomatization and conservative results for fragments of Bounded Arithmetic, Proc. Workshop in Logic and Computation (1987), Contemporary Mathematics AMS, vol.106 (1990), pp 57-84

[6] F.Ferreira; Polynomial Time Computable Arithmetic and Conservative extensions. Ph.D dissertation, Pennsylvania State University, (1988).

[7] P.Hajek, P.Pudlak; Metamathematics of first order arithmetic, to app. in Springer-Verlag.

[8] J.Krajicek; No counter-example interpretation and interactive computation. To appear in: Logic in Computer science, ed. 287-293.

[9] J.Krajicek; Fragments of Bounded Arithmetic and bounded query classes. To appear in the Transactions of AMS.

[10] J.Krajicek, P.Pudlak, and G.Takeuti; Bounded Arithmetic and the polynomial hierarchy, Annals of Pure and Applied Logic 52 (1991), pp 143-153.

[11] J.Paris, A.Wilkie, A.Woods; Provability of the pigeonhole principle and the existence of infinitely many primes. Journal of Symbolic Logic 53, (1988), pp 12311244.

[12] J.Paris, A.Wilkie; Counting problems in Bounded Arithmetic, in: Methods in Mathematical Logic, LNM 1130, Springer (1985), pp 317-340.

[13] P.Pudlak; Ramsey's theorem in Bounded Arithmetic. To appear.

[14] P.Pudlak; Some relations between subsystems of arithmetic and complexity of computations. In: Logic in Comp. Science, ed.

[15] S.M.Riis; Making infinite structures finite in models of Second Order Bounded Arithmetic, in: Arithmetic, Proof theory and computorial complexity, Oxford university press (1993) pp 289-319.

[16] S.M.Riis; Independence in Bounded Arithmetic; DPhil dissertation, Oxford University (1993) 
[17] S.M.Riis; Count $(q)$ does not imply Count $(p)$; Submitted.

[18] A.Wilkie; A model-theoretical proof of Buss's characterisation of the polynomial time computable functions. Notes. (1985). 


\section{Recent Publications in the BRICS Report Series}

RS-94-23 Søren Riis. Finitisation in Bounded Arithmetic. August 1994, 31 pp.

RS-94-22 Torben Braüner. A General Adequacy Result for a Linear Functional Language. August 1994, 39 pp. Presented at MFPS '94.

RS-94-21 Søren Riis. Count(q) does not imply Count(p). July 1994, 55 pp.

RS-94-20 Peter D. Mosses and Martín Musicante. An Action Semantics for ML Concurrency Primitives. July 1994, 21 pp. To appear in Proc. FME '94 (Formal Methods Europe, Symposium on Industrial Benefit of Formal Methods), LNCS, 1994.

RS-94-19 Jens Chr. Godskesen, Kim G. Larsen, and Arne Skou. Automatic Verification of Real-Timed Systems Using EPSILON. June 1994, 8 pp. Appears in: Protocols, Specification, Testing and Verification PSTV '94.

RS-94-18 Sten Agerholm. LCF Examples in HOL. June 1994, 16 pp. To appear in: Proceedings of the 7th International Workshop on Higher Order Logic Theorem Proving and its Applications, LNCS, 1994.

RS-94-17 Allan Cheng. Local Model Checking and Traces. June 1994, 30 pp.

RS-94-16 Lars Arge. External-Storage Data Structures for PlaneSweep Algorithms. June 1994, 37 pp.

RS-94-15 Mogens Nielsen and Glynn Winskel. Petri Nets and Bisimulations. May 1994, 36 pp.

RS-94-14 Nils Klarlund. The Limit View of Infinite Computations. May 1994, 16 pp. To appear in the LNCS proceedings of Concur' '94, LNCS, 1994.

RS-94-13 Glynn Winskel. Stable Bistructure Models of PCF. May 1994, 26 pp. Preliminary draft. Invited lecture for MFCS '94. To appear in the proceedings of MFCS '94, LNCS, 1994. 